\title{
Cellular Automata Dynamics of Nonlinear Optical Processes in a Phase-change Material
}

\author{
Liwei Zhang $^{1,2}$, Robin F. Waters ${ }^{1}$, Kevin F. MacDonald ${ }^{1 *}$, and Nikolay I. Zheludev ${ }^{1,3}$ \\ ${ }^{1}$ Optoelectronics Research Centre \& Centre for Photonic Metamaterials, University of \\ Southampton, Highfield, Southampton, SO17 1BJ, UK \\ ${ }^{2}$ School of Mathematics and Physics, Anqing Normal University, Anqing 246133, P. R. China \\ ${ }^{3}$ Centre for Disruptive Photonic Technologies \& The Photonics Institute, School of Physical and \\ Mathematical Sciences, Nanyang Technological University, Singapore, 637371 \\ * kfm1@soton.ac.uk
}

Changes in the arrangement of atoms in matter, known as structural phase transitions or phase changes, offer a remarkable range of opportunities in photonics. They are exploited in optical data storage laser-based manufacturing, and have been explored as underpinning mechanisms for controlling laser dynamics, optical and plasmonic modulation, and low-energy switching in single nanoparticle devices and metamaterials. Comprehensive modelling of phase change processes in photonics is however extremely challenging as it involves a number of entangled processes including atomic/molecular structural change, domain and crystallization dynamics, change of optical properties in inhomogeneous composite media, and the transport and dissipation of heat and light, which happen on time and length scales spanning several orders of magnitude. Here, for the first time, we show that the description of such complex nonlinear optical processes in phase change materials can be reduced to a cellular automata model. Using the important example of a polymorphic gallium film, we show that a cellular model based upon only a few independent and physically-interpretable parameters can reproduce the experimentally measured behaviors of gallium all-optical switches over a wide range of optical excitation regimes. In an era of otherwise largely opaque computational modelling techniques, the cellular automata methodology has considerable heuristic value for the study of complex nonlinear optical processes without the need to understand details of atomic dynamics, band structure and energy conservation at the nanoscale. 
Sixty years on from the invention of the laser, light-induced structural phase transitions continue to fascinate the research community: Their study provides insight to the fundamental physics of material properties, transition mechanisms and dynamics, and they have had a transformational impact on society, for example in the guise of optical data storage technologies ${ }^{1,2}$ (CDs and DVDs) and laserbased manufacturing processes, from cutting and welding metals, dielectrics and semiconductors at macroscopic scales, to the growing range of laser-based additive and direct-write micro/nanofabrication techniques ${ }^{3-8}$. In photonics, materials undergoing phase transitions, in particular between states with markedly different optical properties, provide a rich variety of functionalities and emerging opportunities for the exploration, manipulation and technological exploitation of light-matter interactions ${ }^{9-11}$ :

- Chalcogenide semiconductor 'glasses' undergoing optically-induced, non-volatile amorphous-crystalline state transitions have long been the foundation of rewritable data storage,; Lately, among numerous emerging applications of chalcogenides in photonics ${ }^{12-14}$, as optically-addressed phase-change media they have facilitated the realization of optically switchable plasmonic metamaterials and nano-antennas, waveguide modulators, and alldielectric metasurfaces providing high-contrast free-space and guided-wave signal modulation relevant to a variety of communications, imaging and sensing applications, rewritable and active flat optical elements that can dramatically reduce the size and weight of optical systems, and are one of the key material platforms in the burgeoning fields of optical RAM (random access memory) and photonic neuromorphic computing ${ }^{15,16}$ :

Gholipour, et al. presented the first metamaterial all-optic switch with functionality underpinned by chalcogenide phase change - a planar plasmonic metasurface hybridized with a thin film of germanium antimony telluride $\left(\mathrm{Ge}_{2} \mathrm{Sb}_{2} \mathrm{Te}_{5}\right.$ or GST-225) ${ }^{17}$, in which reversible, non-volatile amorphous-crystalline transitions provides near- and mid-IR transmission and reflection modulation contrast ratios of $4: 1$ in devices of substantially sub-wavelength thickness (down to $1 / 27$ of the operating wavelength), with active domains as large as 2000 $\mu \mathrm{m}^{2}$ switched by single nanosecond laser pulses. Karvounis, et al. subsequently demonstrated a nanostructured all-chalcogenide (GST-225) dielectric metasurface in which spectral shifting of resonances brought about through laser-induced crystallization delivers reflectivity and transmission switching contrast ratios of up to 5:1 at visible/near-infrared wavelengths selected by design ${ }^{18}$.

Rudé, et al. have utilized GST-225 to control the propagation of near-infrared surface plasmon-polaritons (SPPs) on a $\mathrm{Au} / \mathrm{SiO} 2$ interface $^{19}$ and of light in silicon photonic waveguides ${ }^{20}$ - in both cases, crystallization of a thin amorphous GST cladding layer induced by sub-microsecond laser pulses increases the GST refractive index and absorption coefficient, thereby inhibiting plasmonic/photonic signal propagation in the waveguides.

Michel, et al. have demonstrated reversible, non-volatile switching of infrared plasmonic (aluminum) antenna array resonances via femtosecond pulse laser-induced switching of a 50 nm GST-326 coating ${ }^{21}$, and 'programmable' phase switching of the coating around individual metamolecules within an array of nanorod resonators using single sub-microsecond laser pulses $^{22}$.

Femtosecond pulses have also been employed by Wang, et al. for 'greyscale' phase switching of GST-225: tailored trains of fs pulses are used to achieve discrete reproducible levels of partial crystallization in diffraction-limited domains of a thin GST film, enabling Gbit/in ${ }^{2}$ (non-binary) optical data storage ${ }^{23}$ and the realization of laser-rewritable flat lenses and holographic optical elements ${ }^{24}$. Active flat optical components have also been realized, for example Ruiz de Galarreta, et al. have demonstrated a beam steering device with no moving 
parts based upon a phase-gradient metasurface hybridized with a thin film of GST, switched by near-UV/VIS (405 $\mathrm{nm}$ ) laser irradiation ${ }^{25}$.

The incremental greyscale 'accumulation' property of chalcogenide phase-change media is essential to their application in photonic neuromorphic (brain-inspired) computing paradigm, wherein, as phase-change cells patterned onto photonic (e.g. silicon nitride) waveguides, they can provide optical neuron and synapse functionalities ${ }^{26}$.

- Similarly, the volatile semiconductor-metal transition in vanadium dioxide, $\mathrm{VO}_{2}$ (occurring at $\sim 68^{\circ} \mathrm{C}$ ), has and continues to be investigated for a wide variety of optically-addressed active plasmonic and photonic applications at visible, IR, THz and microwave frequencies ${ }^{27}$.

The ultrafast dynamics of $\mathrm{VO}_{2}$ 's solid-solid phase transition were first interrogated optically by Becker et $a l .{ }^{28}$ and later by Cavalleri, et al. using a fs optical pump / $\mathrm{x}$-ray probe technique ${ }^{29}$, with results suggesting that in this regime the transition is initiated non-thermally. More recent works have suggested that the ultrafast transition in $\mathrm{VO}_{2}$ involves a transient excited electronic (metallic) state retaining the monoclinic (ground-state) structure ${ }^{30-34}$. Rini, et al. subsequently studied the ultrafast optical response of VO2 nanoparticles ${ }^{35}$, observing a large, light-induced ultrafast enhancement of optical absorption in the near-infrared spectral range. Optical-pump, THz-probe techniques have followed ${ }^{36,37}$. Muskens, et al. have also recently reported on antenna- (localized surface plasmon resonance-)assisted picosecond control of nanoscale phase transitions in $\mathrm{VO}_{2}$, with switching energies 20 times lower and recovery times 5 times faster than in bare $\mathrm{VO}_{2}$ films ${ }^{38}$.

Many of the architectures developed for $\mathrm{VO}_{2}$-plasmonic hybrid and all- $\mathrm{VO}_{2}$ metamaterials/surfaces with switchable resonant reflection/transmission characteristics, and guided-wave switching devices, mirror those of their chalcogenide-based counterparts (above) 39-43. However, in consequence of the volatile nature of the metal-semiconductor transition in $\mathrm{VO}_{2}$, these almost invariably rely upon ambient temperature change rather than optical excitation to induce transitions. Recent exceptions to this rule include, for example: the demonstration by Lei, et al., of a nanoscale memory effect in a gold nanodisk arrays on $\mathrm{VO}_{2}$ excited by ultraviolet optical pulses, wherein plasmon resonance modulation depends strongly on the initial state ${ }^{44}$; and the demonstration of a limiting 'optical diode' based upon the asymmetrically nonlinear transmission characteristic of a nanoscale gold/ $/ \mathrm{VO}_{2}$ bilayer ${ }^{45}$.

- Elemental gallium $(\mathrm{Ga})$ subject to nanoscale, optically-driven 'surface melting' in proximity to the near-ambient bulk melting point $(29.8 \mathrm{C}$ ) or solid-solid transitions among crystalline forms in confined (e.g. nanoparticle) geometries at cryogenic temperatures is the medium for which the terms "phase-change nonlinearity" and "active plasmonic" were coined:

Its ability to provide a gigantic, broadband optical nonlinearity via light-induced structural change (between solid and liquid states with markedly different optical properties, as opposed to the conventional purely electronic mechanism of nonlinear optical response) was first reported by Bennet, et al.: a Ga mirror formed at the tip of a single-mode optical fiber being shown to provide for cross-wavelength light-by-light modulation in the telecommunications C-band at milliwatt power levels ${ }^{46}$. Petropoulos, et al. subsequently harnessed this nonlinearity for passive Q-switching of erbium and ytterbium fiber lasers ${ }^{47}$, achieving a level of performance (a self-starting regime generating $~ 50 \mathrm{~ns}$ pulses with peak powers up to 100 W) equivalent to that of state-of-the-art, narrowband semiconductor saturable absorbers. Recently, Waters, et al. have shown that gallium's phase-change nonlinearity can be resonantly enhanced by an order of magnitude in a photonic metamaterial architecture ${ }^{48}$, to offer high contrast all-optical switching near-IR switching at $\mu \mathrm{W} / \mu \mathrm{m}^{2}$, excitation intensities.

Gallium's phase-change nonlinearity was the basis of the original 'active plasmonics' concept $^{49}$, whereby propagation of SPPs on a metal/dielectric waveguide interface can be 
modulated with high contrast via induced changes in the optical properties of one of the two materials, occurring only within a few nanometers of the surface. Krasavin, et al. demonstrated optical modulation of SPP coupling at a $\mathrm{Ga}$ /dielectric interface using few-ns near-IR pulses to drive transient nanoscale melting of the metal ${ }^{50}$; Vivekchand, et al. demonstrated thermally-tuned control of SPP coupling using a Ga grating ${ }^{51}$.

In Ga nanoparticles, Soares, et al. showed that phase-change nonlinearity could facilitate single-particle optical gating and memory functionalities among several different structural forms at few-pJ optical pulse switching energies ${ }^{52,53}$, and this polymorphism is drawing renewed attention in the context of UV plasmonics ${ }^{54,55}$.

Depending upon incident fluence and photon energy, target size/shape/structure, and ambient conditions, optically-induced transitions in any material may proceed through a combination of thermal and/or non-thermal mechanisms on electronic/atomic to macroscopic length scales at femtoto millisecond timescales. Optical measurements, which are based predominantly on interactions between photons and valence or delocalized electrons, cannot directly resolve atomic-scale mechanisms of structural transition. Ab initio computational methods, like density functional theory, molecular dynamics, kinetic equations, or combinations thereof can provide insight to behaviors and mechanisms at the atomic level ${ }^{56,57}$ but are computationally demanding and typically cannot span the full range of length and/or timescales involved. Similar constraints apply to numerical, finite element and finite difference time domain, multiphysics simulations. Alternative approaches to describing phase transition processes, capable of encompassing disparate length/timescales are offered by rate equations $^{58,59}$, phase field methods ${ }^{60,61}$, Monte Carlo models ${ }^{62}$ and, as considered here, cellular automata $(\mathrm{CA})^{63-68}$. Indeed, CA have been applied to modelling dynamics in a remarkably diversity of complex systems, from laser emission to the growth of snowflakes and from ionic diffusion in concrete to pattern recognition in networks ${ }^{69-72}$, as well as the melting and solidification (crystal nucleation/growth/dissociation) of materials ${ }^{63-68}$. However, when considering nominally laser-driven structural transitions, these models tend simply to assume the existence of a heat source, i.e. disregard non-thermal excitation and feedback between the induced change in material properties and the rate of energy deposition. CA models are rarely encountered in the field of optics and photonics because the propagation of light and many of its interactions with matter are very well described either analytically or, for example in complex nanostructured media, via finite element and finite different time domain 'Maxwell solver' numerical simulation techniques. Some exceptions to this rule are found in the domain of quantum/optical computing (esp. quantum dot cellular automata ${ }^{73-78}$ ), occasionally in the study of photonic dynamics (e.g. laser level populations ${ }^{69}$, frequency comb spectral perturbations ${ }^{79}$, collective behaviors in VCSEL arrays ${ }^{80}$ ), and in adaptation of the classic 'Game of Life' evolutionary CA model to account for the retrieval of energy from an environment filled by an electromagnetic field ${ }^{81}$ (leading to behaviors such as light-induced self-structuring and genetic selection). While the numerical methods mentioned above can encompass 'multiphysics' (e.g. light-induced heating) they rapidly become opaque in their complexity and computationally prohibitive where disparate length and time scales are involved and where dynamic structural changes have a strong and nonlinear effect on optical properties. Here we apply a CA methodology for the first time to photo-induced structural transitions in and the associated instantaneous nonlinear optical response of a metal, specifically gallium as a medium in which a strong non-thermal component of response is known to manifest under certain regimes of excitation. The approach offers high heuristic value in its simplicity - the model being defined by a small set of material parameters and others relatable to experimental observables, and practicality in being adaptable to a wide variety of material systems and nano- to macroscopic geometries.

Gallium is an unusually polymorphic element, with several structural phases that have properties ranging from those of the liquid, which is a highly reflective, near-ideal free-electron metal at optical frequencies $^{82,83}$, to those of the stable bulk crystalline form - known as $\alpha$-gallium, which is 
considerably less "metallic" in character (less reflective, more absorbing) ${ }^{84,85}$. $\alpha$-gallium has a structure in which molecular and metallic properties coexist: some inter-atomic bonds are covalent, forming $\mathrm{Ga}_{2}$ dimers and giving rise to a broad optical absorption band extending from 310 to 1820 $\mathrm{nm}$, while the rest are metallic ${ }^{84,86}$. It also manifests "surface melting" ${ }^{87}$, whereby a thin ( few nm) layer of metallic gallium is formed between the solid $\alpha$-phase and a dielectric even at temperatures several degrees below $T_{m}$. The thickness of this interfacial layer, and thereby the reflectivity of the interface, can be controlled in a continuously tunable fashion by low-intensity laser illumination. These characteristics together make gallium a uniquely intriguing and richly functional photonic material but also one in which it is difficult to interpret or disentangle underlying response mechanisms. The dynamics of light-induced reflectivity changes at gallium/dielectric interfaces, and their dependence upon intensity or fluence and the temperature of the bulk metal, have been studied under various regimes of pulsed optical excitation ${ }^{88-90}$ : At the shortest (fs to ps) timescales, nonequilibrium electronic excitation dominates the reflective response; at longer timescales, thermal effects (i.e. laser-induced heating) alone are insufficient to account for observed dependences of induced reflectivity change on temperature and a substantive non-thermal contribution to interfacial metallization (based upon the direct optical excitation of the dimer covalent bonds in $\alpha$-gallium) is inferred. We find here that reflectivity dynamics at a gallium-dielectric interface are elegantly described for the full range of fs to $\mu$ s pulse durations by a simple 3-level cellular automata model including a non-thermal excitation channel, operating under a minimal set of transition rules that are invariant with pulse duration.

Cellular automata are fully discrete dynamic systems, wherein the state of each cell in a regular $n$ dimensional lattice is chosen from a finite set of possible states and temporal evolution runs synchronously in all cells ${ }^{91,92}$. Each cell evolves in each time step according to a set of transition rules dependent upon its initial state and the those of other cells within a defined (e.g. von Neumann, Moore) neighborhood. Here we have constructed a two-dimensional CA model of minimally sufficient complexity (see Methods) to describe the photo-induced metallization dynamics of Ga at a planar gallium-silica interface: Each gallium cell (Fig. 1a) can exist in one of three states (Fig. 1b) a lowest-energy 'ground' state (Level 1), a higher-energy 'metallic' state (Level 2) and a highestenergy, short-lived 'optically excited' state (Level 3). The ground state possesses optical properties identical to those of $\alpha$-gallium ${ }^{85}$, while the optically excited state and metallic states possess the optical properties of liquid gallium ${ }^{82} . \Gamma_{13}$ is the optical pumping rate driving excitation from the ground state (1) to the excited state (3) and depends upon the incident photon flux, i.e. incident light intensity, which in turn is a function of time $t$, i.e. pulse duration and profile. $\Gamma_{12}$ is the rate of thermally-induced transition from the ground state (1) to the metallic state (2) and depends upon the proximity of local temperature to $T_{m}$. As such, it is a function of both ambient temperature (tending exponentially to one as $T \rightarrow T_{m}$ and to zero as $\left[T_{m}-T\right]$ increases) and light-induced temperature change (i.e. pulse duration, profile and fluence). $\gamma_{32}$ and $\gamma_{21}$ are respectively the decay probabilities from the excited state (3) to metallic state (2) and from the metallic state (2) to the ground state (1) within a single time step $\delta t$ (see Supplementary Fig. S1).

The evolution of each cell in each time step is governed by a set of four rules:

- Rule 1: Cell in the GROUND state become EXCITED if they absorb a photon (the probability of absorption being a function of the optical skin depth - see Supplementary Information).

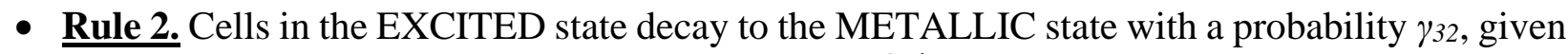
by the lifetime of the excited state $\tau_{3}: \gamma_{32}=1-e^{-\delta t / \tau_{3}}$.

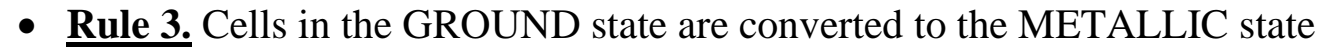

$\circ$ with a probability $\Gamma_{12}$ when the number of neighboring cells (among the eight surrounding, i.e. nearest and next-nearest) already in the METALLIC or EXCITED state is greater than 1 and less than or equal to a threshold $N_{12}$; 


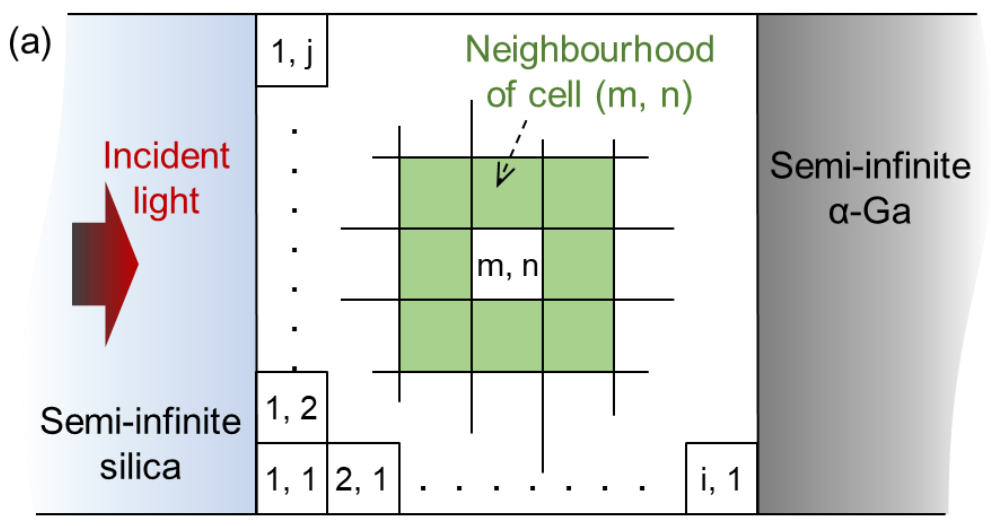

(b) Level 3: Optically excited

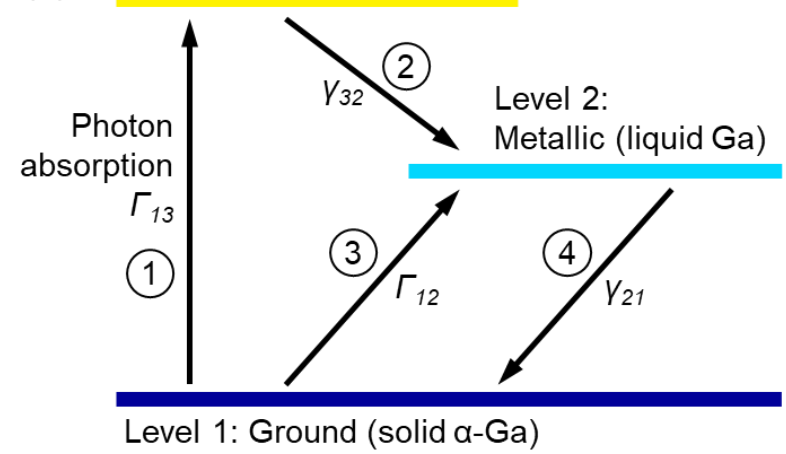

Fig. 1. Cellular automata model of a gallium/silica interface. (a) Schematic of the 2D cellular array and the adjacent semi-infinite silica and solid gallium volumes assumed to be present in the evaluation of interface reflectivity. Each Ga cell can exist in one of three states as shown in panel (b): a lowest-energy 'ground' state a higher-energy metallic state; and a highest energy, short-lived 'optically excited' state. (b) Threelevel system of gallium cell states and associated transitions, annotated with transition probabilities $[r, \gamma]$ and accompanying CA transition rule numbers [circled].

○ with certainty (probability $=1$ ) when the number of METALLIC or EXCITED neighbors is $>N_{12}$.

- Rule 4. Cells in the METALLIC state decay to the GROUND state with a probability $\gamma_{21}=$ $1-e^{-\delta t / \tau_{2}}$, where $\tau_{2}$ is the lifetime of the metallic state, when the number of METALLIC or EXCITED neighbors is less than or equal to a threshold $N_{21}$.

Rules 1 and 2 describe the non-thermal photo-induced metallization process, whereby the absorption of a photon leads to the breaking of a $\mathrm{Ga}-\mathrm{Ga}$ dimer bond and the structural reconfiguration of the associated unit cell in the atomic lattice. Rules 3 and 4 describe the balance between metallization (c.f. melting) and recrystallization of cells based effectively upon the transfer of absorbed energy from electrons to the lattice and from cell-to-cell through the lattice; and/or upon changes in the energetically-preferred configuration of ground state cells surrounded by numerous metallic cells and vice-versa. (Schematic dependences of transition probabilities on neighborhood are shown in Supplementary Fig. S2.)

The lifetimes of the excited and metallic states are set respectively at $\tau_{3}=1 \mathrm{ps}$ and $\tau_{2}=1 \mathrm{~ns}$. Exact values for gallium are not known so these are representative, order-of-magnitude values ${ }^{93}$ sufficient to the present purpose of a self-consistent comparison among metallization dynamics across a range of pulsed optical excitation regimes. Indeed, we specifically do not aspire to quantitatively replicate any particular set of prior experimental data, and as results will illustrate (below) it is relative rather 
than absolute values of lifetimes and pulse duration that determine behaviors within the CA model. A third time constant of importance is the 'thermalization time' $\tau_{t}$, over which heat is dissipated from the region in which it is generated through photon absorption. This nonlinear heat flow (dependent upon both spatial and temporal pulse profile, sample structure and ambient temperature) can lead to complex reflectivity relaxation dynamics ${ }^{88,89}$. For the CA model we assume representative, suitably physical, order-of-magnitude value $\tau_{t}=1 \mu \mathrm{s}$.

\section{Results and discussion}

We first consider the short pulse excitation regime, with a CA model time step $\delta t$ of 1 fs and (pump) pulse duration $\tau_{p}$ of 100 steps, i.e. $100 \mathrm{fs}$, centered at time step 150. Figure 2 a shows the dynamics of interface reflectivity at the $\lambda=775 \mathrm{~nm}$ pump wavelength (c.f. frequency degenerate pump-probe measurements) for a range of excitation fluences, with neighbor threshold values $\mathrm{N}_{21}, \mathrm{~N}_{12}=5$ and a transition probability $\Gamma_{12}=8 \times 10^{-5}$ (see Supplementary Information). In general, $\Gamma_{12}$ is a function of incident fluence (and therefore time during a Gaussian excitation pulse) and temperature but in the femtosecond regime it can be taken as constant during and considerably beyond the pulse duration because $\delta t, \tau_{p}<<\tau_{2}, \tau_{t}$. Reflectivity dynamics are correspondingly dominated by the photo-excitation channel. For the same reason, the values of $N_{12}$ and $N_{21}$ are found to be of negligible consequence to response dynamics within the first several ps during and after a fs pulse (see Supplementary Fig. S4), which is to say that on such timescales the state of each cell evolves independently, without influence from its neighbors.

Reflectivity dynamics are characterized, as in corresponding experimental studies ${ }^{89,90}$, by a sharp increase in reflectivity during the excitation pulse, to a level that increases with fluence, followed by a much longer and shallower rise over picosecond timescales after the pulse as shown in Fig.2(b). Snapshots of phase state distribution at representative time intervals are presented in Figs. 3(a)-(h) [a full video file of phase state evolution with single time-step resolution is available online] show that
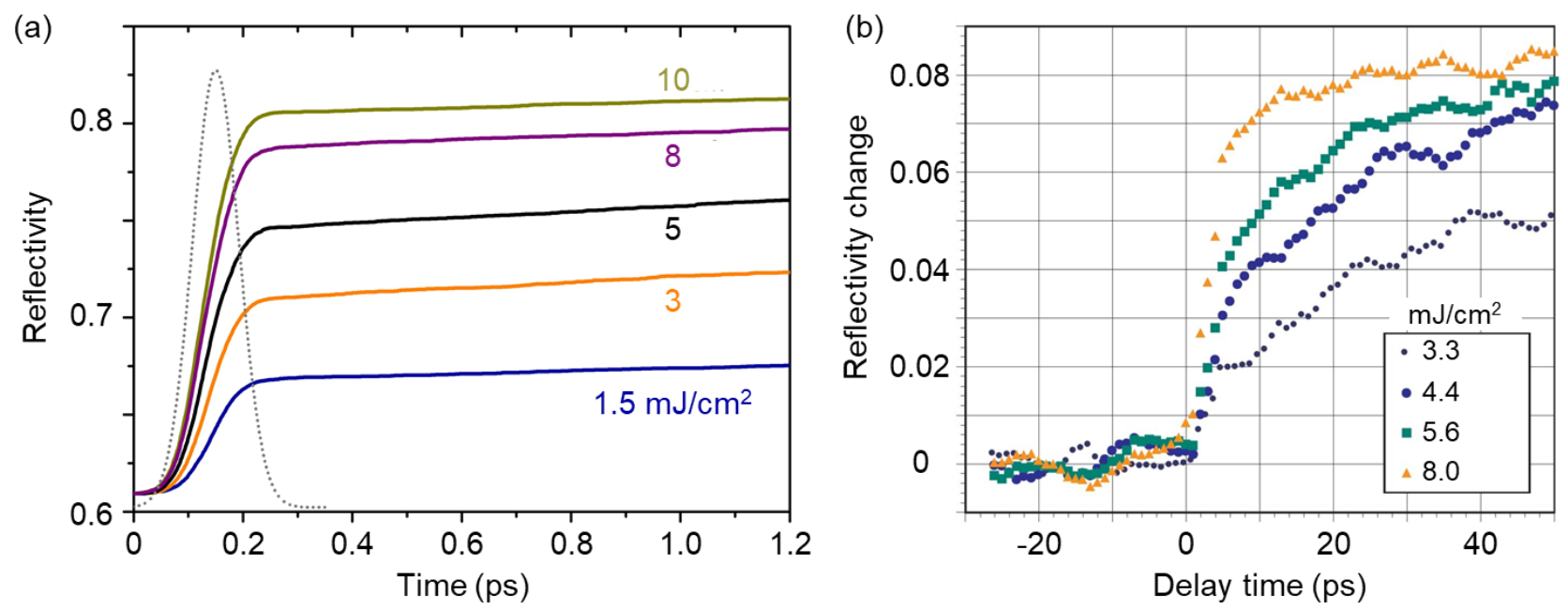

Fig. 2. Reflectivity dynamics in the short pulse excitation regime. (a) CA modelled Ga-silica interface reflectivity at $775 \mathrm{~nm}$ as a function of time [time step $\delta t=1 \mathrm{fs}$ ] during and immediately after $100 \mathrm{fs}$ pump pulses of varying fluence [as labelled; $N_{12}, N_{21}=5 ; \Gamma_{12}=8 \times 10^{-5}$ ]. The dashed grey line shows the Gaussian temporal profile of the excitation pulse [normalized amplitude - vertical axis scale does not apply]. (b) From Ref. 89, experimentally measured Ga-silica interface reflectivity at $800 \mathrm{~nm}$ as a function of time around 150 fs pump pulses of varying fluence [as labelled]. Note that the full $1.2 \mathrm{ps}$ span of panel (a) corresponds to a small fraction of the axial range in (b). 
(a)

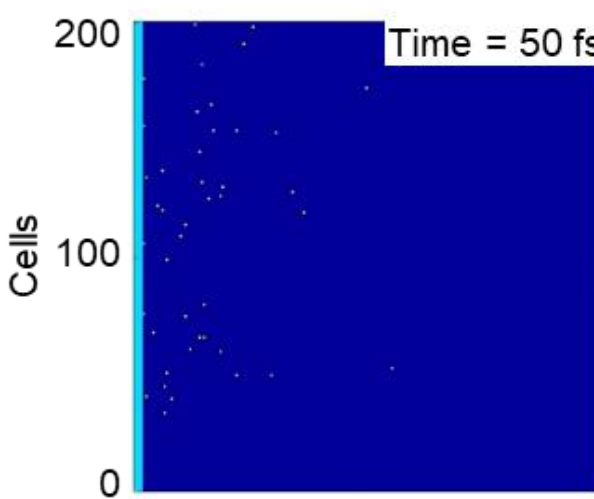

(c)

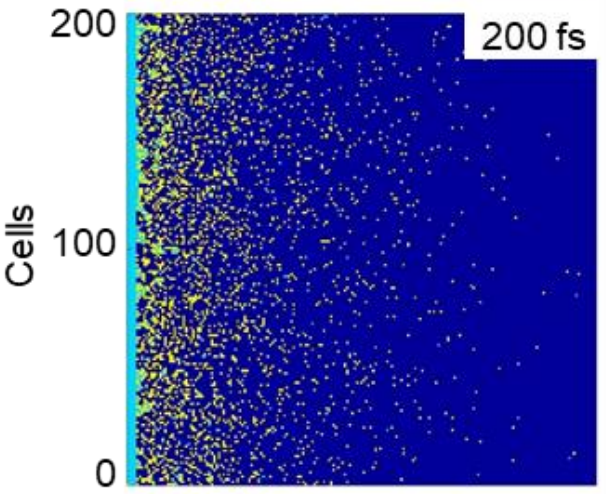

(e)

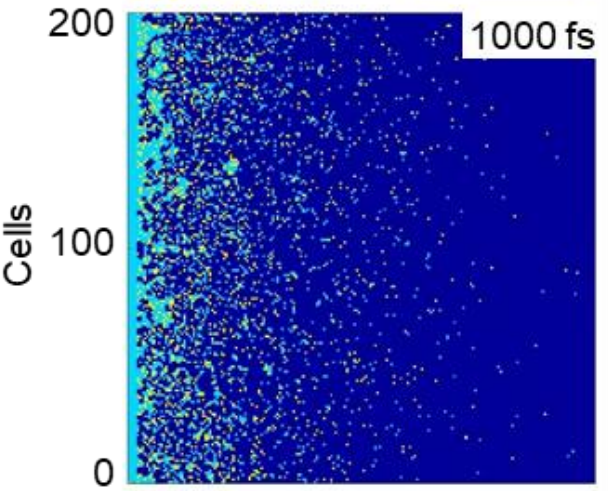

(g)

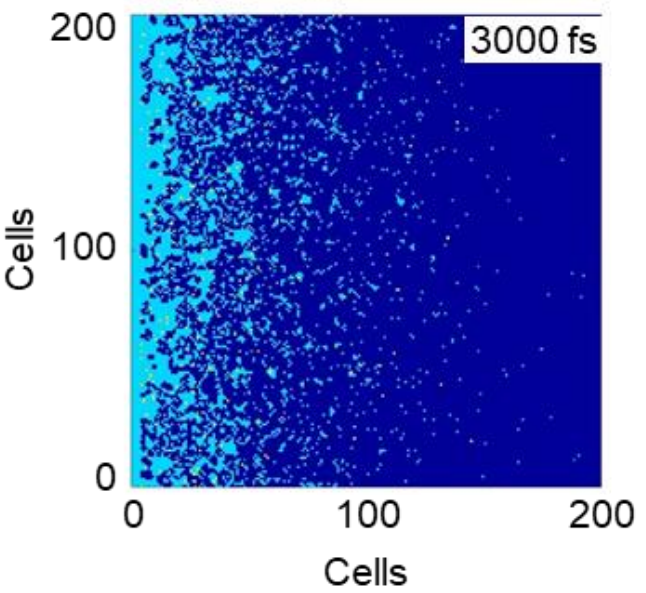

(b)

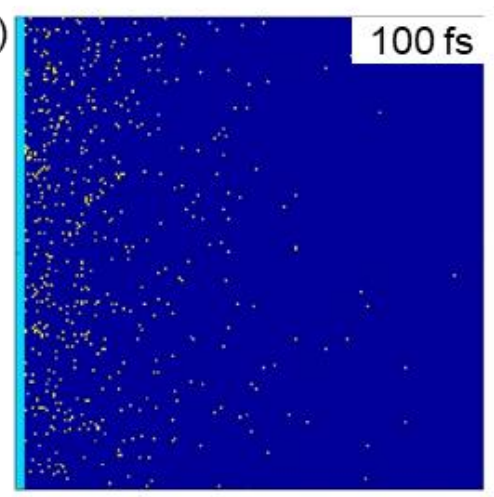

(d)

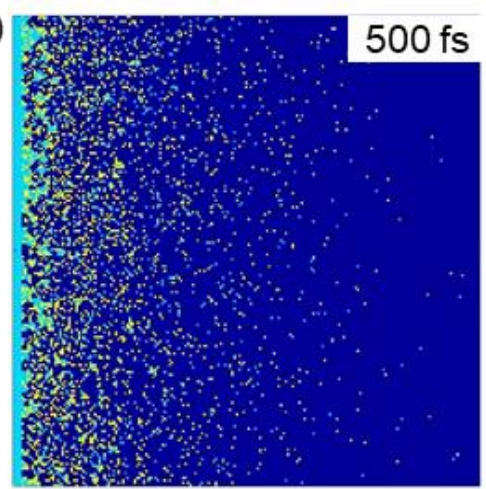

(f)

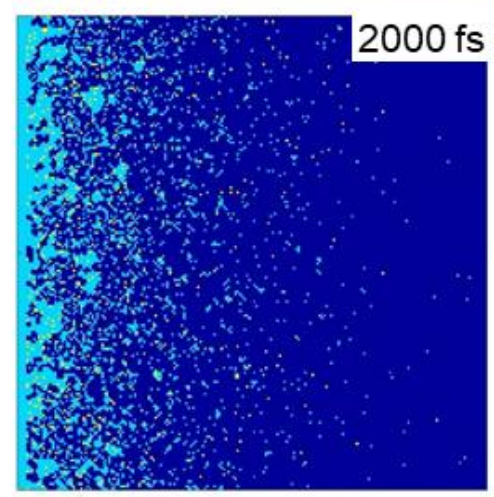

(h)

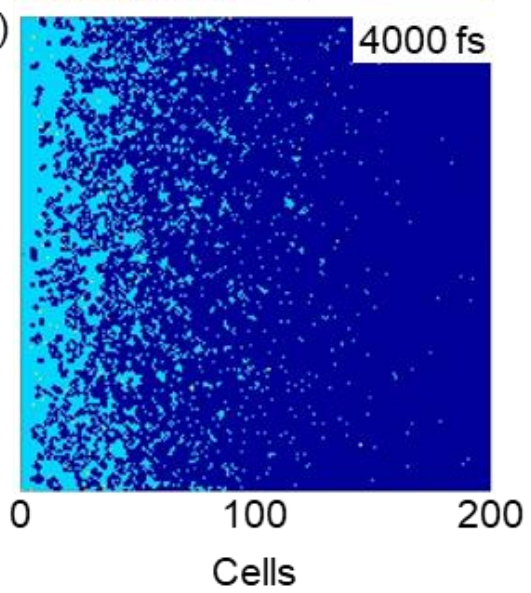

Ground

Optically excited

Metallic

- $10 \mathrm{~nm}$

Fig. 3. Temporal evolution of phase-state distribution among cells in the short pulse excitation regime. Representative maps of cellular phase state (ground, optically excited or metallic) at intervals of between 50 and 4000 time steps [as labelled, step $\delta t=1 \mathrm{fs}$ ] around a $100 \mathrm{fs}$ pump Gaussian pulse of fluence $F_{i n}=5$ $\mathrm{mJ} / \mathrm{cm}^{2} .\left[N_{12}, N_{21}=5 ; \Gamma_{12}=8 \times 10^{-5}\right.$. The silica interface is at the left-hand side of each panel and light is incident from that direction. [Multimedia view] 
this is a consequence of a rapid (non-thermal) increase in the population of cells in the EXCITED state during the pulse. These are randomly distributed with a number density that decreases exponentially, as one would expect, with distance from the gallium/glass interface. These transition to the METALLIC state and thermal (neighbor interaction) mechanisms then dominate on picosecond timescales and beyond, sustaining an upward trend in reflectivity (based on Rule 3) for some time after the pulse. Note here that at all points in the gallium bulk (beyond the persistent surface-melt layer at the glass interface), melting is 'incomplete' even at long time intervals after the excitation pulse - there is no continuously molten layer of defined fluence-, temperature- and time-dependent depth. The structure of METALLIC inclusions in a GROUND state matrix (or vice-versa close to the interface) seen in the CA model is consistent with prior analytical inference of fractional light-induced melting from reflectivity measurements in the fs-pulsed excitation regime ${ }^{90}$.

We now consider the very substantial effect of pulse duration on metallization dynamics. In the CA model this is achieved by maintaining a fixed pulse duration $\tau_{p}$ of 100 time steps (centered at step 150) while changing the step size $\delta t$, in the present case from $1 \mathrm{fs}$ to $10 \mathrm{~ns}$ in seven order-of-magnitude increments. We maintain near-neighbor threshold values $N_{12}, N_{21}=5$ and assume a fixed fluence of $F_{\text {in }}=5 \mathrm{~mJ} / \mathrm{cm}^{2}$ across all pulse durations.

Figures $4(\mathrm{a}-\mathrm{h})$ show snapshot phase state distributions at the $150^{\text {th }}$ time step, i.e. at the peak of the pulse when photon flux is at maximum, for pulse durations ranging from 100 fs to $1 \mu \mathrm{s}$ (A corresponding set of snapshots for the $250^{\text {th }}$ time step, in the tail of the pulse, are shown in Fig. S5). For short pulse durations $\leq 1 \mathrm{~ns}$ (Fig. 4a-e), i.e. shorter than the metallic state lifetime $\tau_{2}$, there is a diffuse population of cells in the optically excited and/or metallic state. This extends, with decaying number density, over several skin depths into the gallium bulk with almost no change in the thickness of the persistent surface-melt layer at the glass interface. There is a significant proportion of excitedstate cells only for pulse durations $\leq 10$ ps (Fig. $4 \mathrm{a}-\mathrm{c}$ ), i.e. while the time step $\delta t$ remains shorter than the excited state lifetime $\tau_{3}$ (for durations $>10$ ps but $\leq 1 \mathrm{~ns}$, cells excited in a given time step will transition to the metallic state with near-certainty in the next).

This diffuse, non-equilibrium (non-thermal) distribution of excited/metallic cells is responsible for the two-stage, fast and then much slower, interface reflectivity dynamic that is characteristic of shortpulse excitation regimes (as illustrated in Supplementary Fig. S6a-e). It is entirely absent for pulse durations $\geq 100 \mathrm{~ns}$ (Fig. 4g-h) because any isolated metallic cells revert to the ground state with near certainty $\left(\gamma_{2 l} \rightarrow 1\right)$ in every time step. Cells can only be sustained in the metallic state for more than one time step by the neighbor rules, which is to say thermally. This leads to contiguous 'growth' of the surface-melt layer into the gallium bulk and a proportionate, steady increase in interface reflectivity over the duration of the pulse (Fig. S6g-h).

Figure 4(f), for a pulse duration of $10 \mathrm{ns,} \mathrm{shows} \mathrm{characteristics} \mathrm{of} \mathrm{both} \mathrm{the} \mathrm{short-} \mathrm{and} \mathrm{longer-pulse}$ regimes: i.e. both a not-insignificant population of individual/clustered metallic-state cells within the ground-state bulk and a clear change in the thickness of the interfacial surface-melt layer. Reflectivity dynamics (Fig. S6f) are correspondingly mixed, showing elements of both a fast initial increase and a slower, steady change over the pulse duration. This intermediate case illustrates how the boundary between thermal and non-thermal melting regimes is predominantly a function of metallic state lifetime $\tau_{2}$ (assuming that $\tau_{3}$ will always be $<<\tau_{2}$ ).

In the long pulse $(\geq 100 \mathrm{~ns})$ regime, once state lifetimes $\left(\tau_{2}, \tau_{3}\right)$ and neighbor thresholds $\left(N_{12}, N_{21}\right)$ are set, reflectivity dynamics for a given fluence become a function solely of $\Gamma_{12}$, the local-temperaturedependent probability of transition from the ground to the metallic state. For square optical pulses shorter than the thermalization time $\left(\tau_{p}<\tau_{t}\right)$ incident on a sample at a fixed ambient (bulk gallium) temperature, $\Gamma_{12}$ can be taken as constant over the pulse with a value effectively proportional to the magnitude of light-induced temperature change. In this way - by varying $\Gamma_{12}$, with a fixed time step $\delta t$ of $1 \mathrm{~ns}$, near-neighbor thresholds $N_{12}, N_{21}$ again equal to 5, the CA model is able (Fig. 5) to 
(a)

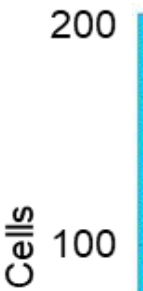

(c)

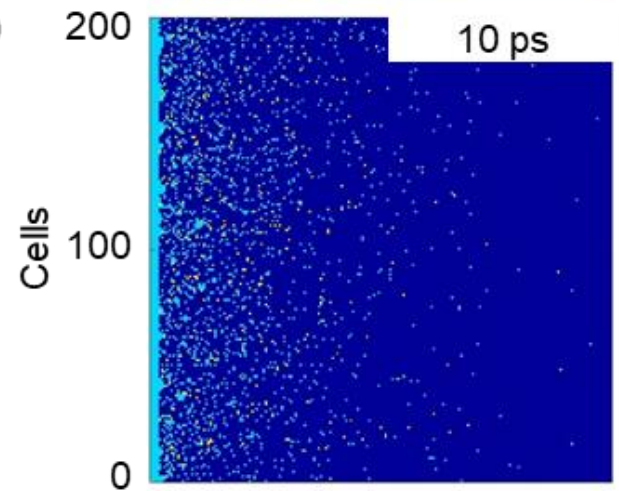

(e)

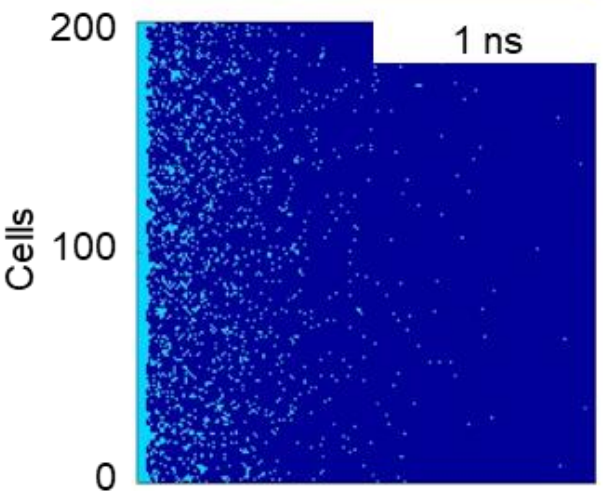

(g)

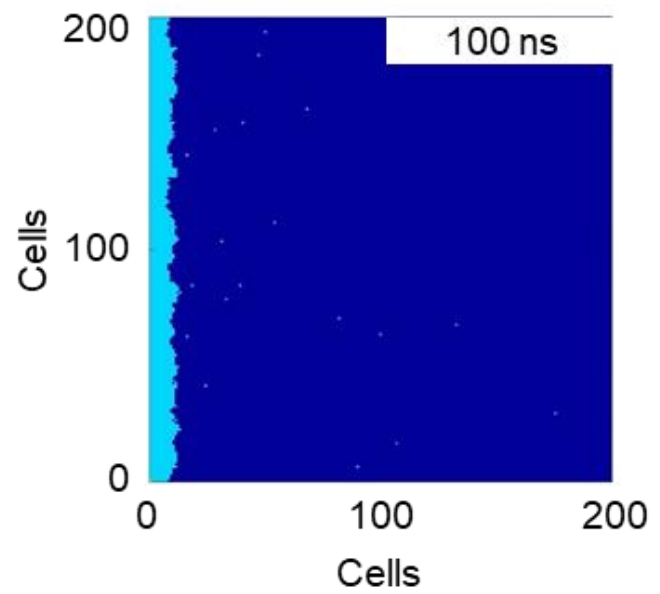

(b)

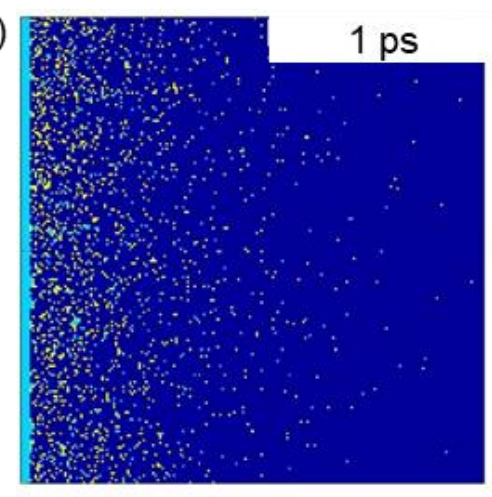

(d)

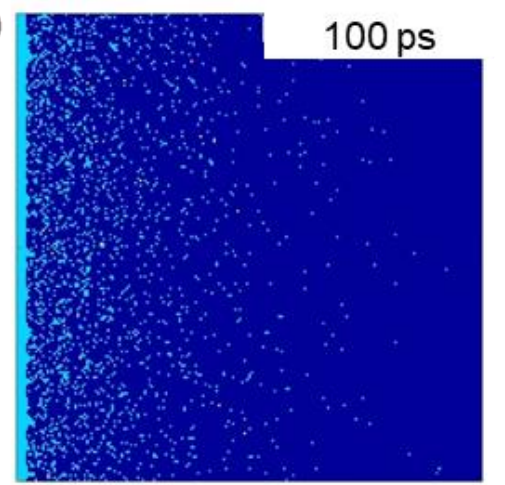

(f)

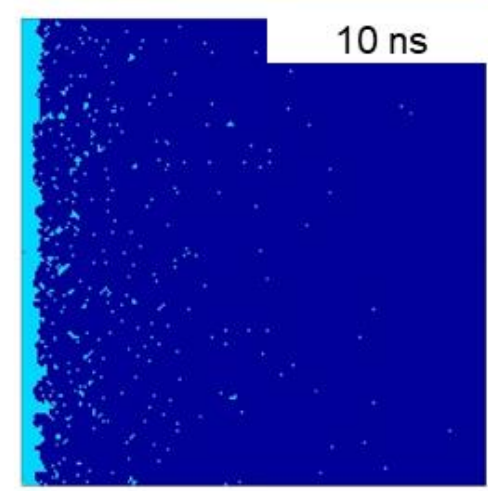

(h)

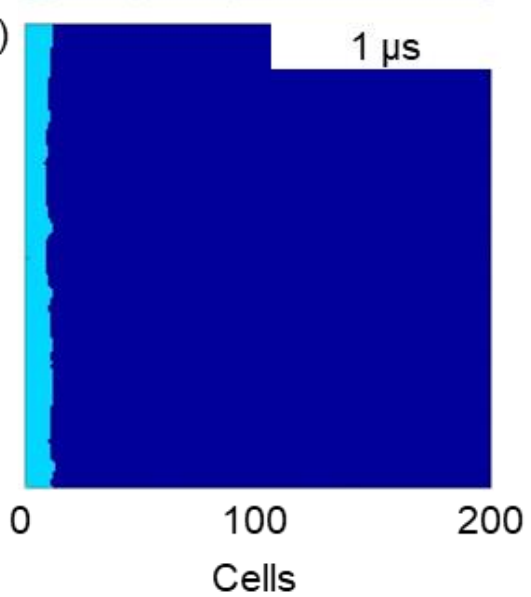

Ground

Optically excited

Metallic

- $10 \mathrm{~nm}$

Fig. 4. Phase-state distribution as a function of pulse duration. Representative maps of cellular phase state at the peak of Gaussian excitation pulses with durations $\tau_{p}$ ranging from $100 \mathrm{fs}$ to $1 \mu \mathrm{s}$ [as labelled] in orderof-magnitude increments. $\left[F_{i n}=5 \mathrm{~mJ} / \mathrm{cm}^{2} ; N_{12}, N_{21}=5 ; \Gamma_{12}=8 \times 10^{-5}, 1.2 \times 10^{-4}, 1.5 \times 10^{-4}, 5.2 \times 10^{-4}, 5.25 \times 10^{-3}\right.$, $5.65 \times 10^{-2}, 0.465$ and 0.9 respectively.] 
qualitatively reproduce the experimental results of Ref. 88. In experiment, the local temperature is a function of incident fluence but in the CA model the two are decoupled: photon flux alone - the value of $F_{\text {in }}$ - does not affect the effective temperature, which is encapsulated in $\Gamma_{12}$. Thus, for the purposes of this analysis we maintain a fixed value of $F_{\text {in }}$ and only change $\Gamma_{12}$. Reflectivity (c.f. metallized layer thickness) increases monotonously with time over the pulse duration at a rate that increases with the value of $\Gamma_{12}$, i.e. with induced local temperature change (in experiment, with peak incident power); The dependence of reflectivity on time is (near-)linear at lower values of $\Gamma_{12}$ but saturates at higher values as the metallized layer thickness tends towards the optical skin depth. (The latter behavior is not seen in the experimental case simply for lack of laser power.)

In contrast to the fs-pulse regime, where they are of negligible consequence, the neighbor thresholds do influence reflectivity dynamics in the long-pulse thermal regime (as shown in Supplementary Fig. S7) - the effect of changing $N_{12}$ is minor but the value of $N_{21}$ has a more significant impact. These thresholds are effectively a coarse surrogate for ambient temperature, with lower values corresponding to bulk Ga temperatures closer to $T_{m}$, whereby a given fluence can induces a larger change in reflectivity.

When optical excitation is withdrawn, metallic state cells relax to the $\alpha$-Ga ground state (i.e. recrystallize) and interface reflectivity correspondingly recovers to its pre-excitation level. This process is governed by the complex, nonlinear dynamics of heat flow and in practice will be a function of pulse duration and spatial and temporal intensity profile, sample structure and the proximity of ambient (silica and Ga bulk) temperature to $T_{m}$. For the purposes of the CA model, characteristic experimentally observed reflectivity relaxation behaviors can be qualitatively reproduced (Fig. 5b) by simply stepping down the value of $\Gamma_{12}$ at the end of an excitation pulse. Recall here that this transition rate is essentially a function of local temperature: As such, lower post-excitation values $\Gamma_{12}$ ' can be taken denote lower ambient temperatures (i.e. higher values of $T_{m}-T$ ). Under the course approximation of a step-function in $\Gamma_{12}{ }^{(\text {) }}$ and with the same value of transition probability applying to all cells in the array, the CA model does not replicate the exponential form of the decay curves seen in experiment (Fig. 5c). However, it nonetheless sufficient to illustrating that for a given set of excitation conditions, lower ambient temperatures promote faster recrystallization and reflectivity relaxation.

\section{Summary}

In summary, we show here that a cellular automata (CA) model - a three-level system governed by only four transition rules and a sparse set of independent material and process parameters - can phenomenologically describe the complex, non-stationary, spatially inhomogeneous dynamics and resulting nonlinear optical properties of a medium undergoing a light-induced structural phase transition.

We consider the case of solid gallium near to its bulk melting transition, as a non-trivial system (in which optical excitation may include a strong non-thermal component and surfaces are subject to nanoscale 'pre-melting') of relevance to a variety of photonic (all-optical and 'active plasmonic') switching applications. Non-equilibrium behaviors in systems close to a transition point cannot be described analytically and they present considerable challenges in computational (e.g. multiphysics finite element) simulation, particularly in regard to the combination of macro- and nanoscopic length scales. In this context it is remarkable that a CA model (which notably does not include local or ambient temperature as an independently defined parameter) is able to reproduce light-induced transition and relaxation dynamics over seven orders of excitation pulse duration magnitude (from femto- to microsecond) and provide insight to the microscopic mechanisms of transition without recourse to deep understanding or analytical description of atomic dynamics, band structure, electromagnetics, thermodynamics or nanoscale energy conservation. It emerges clearly from the CA 
(a)

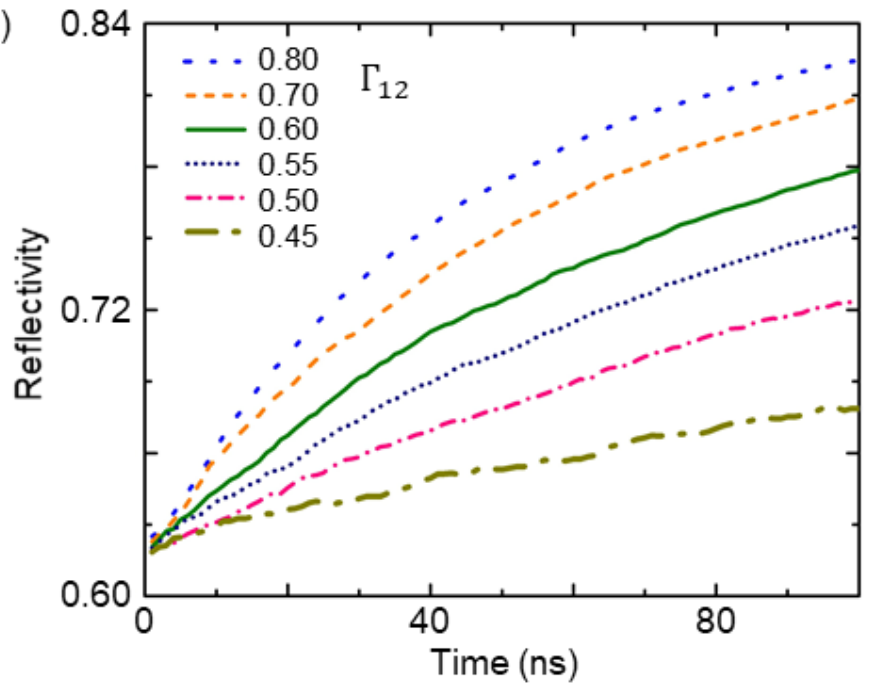

(b)

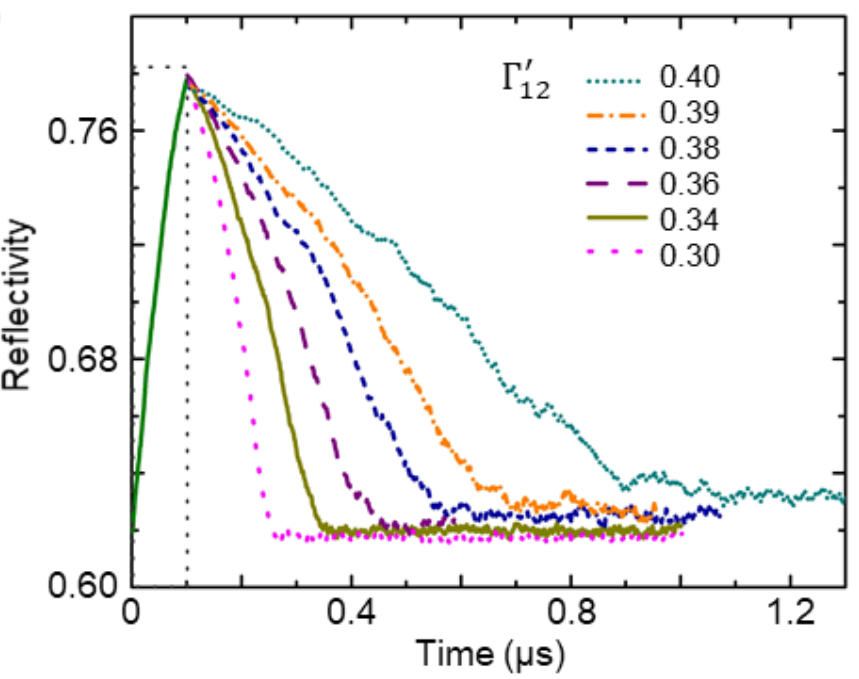

(c)

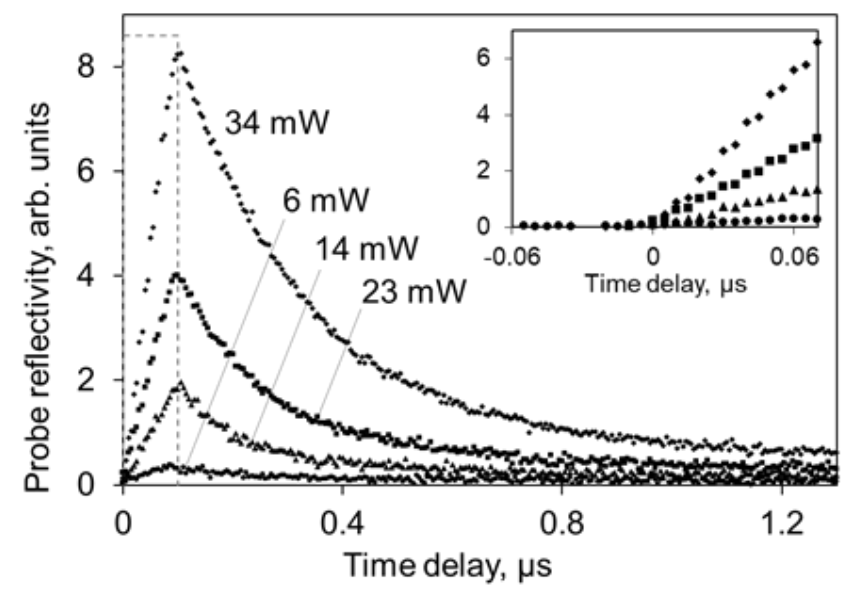

Fig. 5. Reflectivity dynamics in the long pulse excitation regime. (a) CA modelled Ga-silica interface reflectivity at $775 \mathrm{~nm}$ as a function of time [time step $\delta t=1 \mathrm{~ns}$ ] during $100 \mathrm{~ns}$ pump pulses, $\Gamma_{12}$ increase from 0.45 to 0.8 . [ $\left.F_{i n}=5 \mathrm{~mJ} / \mathrm{cm}^{2} ; N_{12}, N_{21}=5\right]$. (b) Interface reflectivity as a function of time after a $100 \mathrm{~ns}$ pump pulse [ $\Gamma_{12}=0.6 ; N_{12}, N_{21}=5$ ] for a selection of post-excitation transition probabilities $\Gamma_{12}{ }^{\prime}$ [as labelled]. The dashed black line shows the square temporal profile of the excitation pulse [normalized amplitude vertical axis scale does not apply]. (c) After Ref. 88, experimentally measured Ga-silica interface reflectivity at $1550 \mathrm{~nm}$ as a function of time during and after excitation with $100 \mathrm{~ns} 1536 \mathrm{~nm}$ pump pulses of varying peak power [as labeled] at a fixed ambient temperature of $24^{\circ} \mathrm{C}\left(T_{m}-T \sim 5^{\circ} \mathrm{C}\right)$. The inset shows dynamics near the rising edge of the pump pulse on an expanded time scale. 
model that transition and reflectivity dynamics in the Ga system are controlled predominantly by the relative values of optically-excited and metallic state lifetimes. These determine whether thermal or non-thermal mechanisms dominate and thereby whether the metallization of a-Ga is diffuse (in the short, fs-ps pulse regime) or proceeds through the motion of a defined solid-liquid melt front (longer pulses). Particularly interestingly, the CA model also shows that neighborhood is essentially irrelevant in the short pulse excitation regime: at fs timescales there is no coupling between $\mathrm{Ga}$ (crystalline) cells and each cell responds independently to the flux of incident photons.

We believe that the cellular automata approach may be applied heuristically to a variety phase-change, nonlinear optical and active (nano)photonic systems - for example, the three-level model developed here for $\mathrm{Ga}$ may be adapted to $\mathrm{VO}_{2}$, where ultrafast transitions also appear to involve a transient electronically excited state ${ }^{30-34}$. In reducing complex systems to a minimally sufficient set of rules and parameters, CA models may not produce the most accurate quantitative fit to experiment, but their value lies in the provision of simple insight to system dynamics and the relative importance of physical parameters, which can inform the design of further experiments and more refined models. A two-dimensional cellular array is sufficient here to modelling the optical properties of an effectively infinite planar metal-dielectric interface illumined by a plane wave (i.e. as a generic approximation to the experimental realities of Refs. 88, 89). At the expense of the periodic boundary condition employed in the direction parallel to the interface, the model could readily accommodate inhomogeneous incident beam intensity profiles. Extension to three spatial dimensions would provide for consideration of more complex geometries and finer control of neighbor interaction rules and thresholds (i.e. with 26 surrounding cells in a simple 3D cubic neighborhood as compared to 8 in the 2D square case).

\section{Methods}

Cellular geometry. Our CA model employs a two-dimensional square array of cells with a lattice constant $L_{c}=0.55 \mathrm{~nm}$ (of order the $\alpha$-gallium crystalline unit cell size ${ }^{94}$ ). The model domain has a depth, in the direction of incident light propagation, of 200 cells (approximately 3 times the optical skin depth of $\alpha$-gallium at the assumed near-infrared illumination wavelength) and a width, parallel to the gallium-silica interface, of again 200 cells ( $>20$ times the thermal diffusion length in liquid gallium over $1 \mathrm{ps}$; thermal diffusivity ${ }^{95} \approx 0.2 \mathrm{~cm}^{2} / \mathrm{s}$ ). The positional address of each cell is denoted by coordinates $i$ (depth) and $j$ (width).

Incident photons. We assume incident monochromatic light pulses with either Gaussian or square temporal intensity profiles. In the former case this gives an absorbed intensity profile of the form

$$
I=I_{L}(1-R) e^{-4 \ln 2\left(\frac{t}{\tau_{p}}-1.5\right)^{2}}
$$

where $I_{L}$ is the incident intensity, $R$ is the reflectivity of the $\mathrm{Ga} /$ silica interface, and $\tau_{p}$ is the full-width half-maximum pulse duration. Fluence for a Gaussian pulse ${ }^{96}$ is given by

$$
F_{\text {in }}=I_{L} \tau_{p} \sqrt{\frac{\pi}{4 \ln 2}}
$$

and thereby the number of photons entering each CA row $j$ in a given time step is

$$
n_{0}=\frac{1}{h \tau_{p} c} \sqrt{\frac{4 \ln 2}{\pi}}(1-R) F_{i n} \delta_{t} L_{c}^{2} \lambda e^{-4 \ln 2\left(\frac{t}{\tau_{p}}-1.5\right)^{2}}
$$

where $h$ is the Planck constant and $\lambda$ is the vacuum wavelength of incident light. 
In the case of a square pulse, $F_{\text {in }}=I_{L} \tau_{p}$ and the number of photons per CA row per time step is constant during the pulse: $n_{0}=\frac{1}{h \tau_{p} c}(1-R) F_{i n} \delta_{t} L_{c}^{2} \lambda$.

Under Rule 1, a cell in the ground state may absorb one photon with probability $A b=0.03$ (a value derived from skin depth - see Supplementary Information). A photon not absorbed in cell $i$ passes, within the same time step, sequentially to cells $i+1, i+2$, etc. until it is absorbed. The probability that a photon will pass through all Ga cells without being absorbed is $<0.0025$.

Interface reflectivity. Interface reflectivity $R$ is evaluated in each time step using the transfer matrix method, treating the system as a stack of layers:

- the incident medium is taken to be semi-infinite silica with a relative permittivity $\varepsilon_{\text {silica }}=2.28$ (refractive index 1.51);

- at the interface we assume an ever-present 4 cell $\left(d_{0}=2.2 \mathrm{~nm}\right)$ thick surface melt layer of gallium in the metallic state ${ }^{87}$;

- the rest of the $i=5$ - 200 cell thickness of gallium is divided into six layers - five 10-cell layers for pulse durations $\leq 10^{7}$ fs or 4-cell layers for pulse durations $>10^{7}$ fs (for reasons made apparent in Fig. 4) followed by one 146 / 176 cell layer;

- $\quad$ beyond $i=200$ we assume a semi-infinite thickness of (ground state) $\alpha$-gallium.

Each CA gallium layer is assigned an effective permittivity calculated as a weighted mean of the $\alpha$ and liquid gallium permittivities ( $\varepsilon_{\alpha}$ and $\varepsilon_{\text {liquid }}$ respectively) according to the numbers of cells correspondingly in the ground and excited or metallic states: $\varepsilon_{e f f}=q \varepsilon_{\alpha}+(1-q) \varepsilon_{\text {liquid }}$, where $q$ is the filling fraction of ground state cells.

Neighborhood. We utilize a Moore neighborhood ${ }^{92}$, whereby the evolution of each cell in each time step is influenced by the state of the eight surrounding (four nearest and four next-nearest) cells. The model domain is taken to be periodic (or wrapped) in the $j$ direction such that all cells have a full complement of eight neighbors, i.e. rows $(i, 1)$ and $(i, 200)$ are adjacent to one another.

The neighbor number thresholds $N_{12}$ and $N_{21}$, applied under Rules 3 and 4 , must take values $\geq 5$ because lower values are unphysical: in the case of $N_{12}$ they can lead to self-sustaining chains of cell conversion adjacent to the persistent surface-melt layer or indeed any column $i$ of all metallic- or excited-state cells; In the case of $N_{21}$, they would enable adjacent full columns of metallic-state cells to remain indefinitely in that state.

\section{Supplementary Material}

See supplementary material for:

- The dependences of transition probabilities on CA model time step and neighborhood;

- The calculation of photon absorption probability per cell;

- Evaluation of the threshold value of $\Gamma_{12}$;

- The effect of neighbor threshold values $N_{12}, N_{21}$ on reflectivity dynamics (in short and long pulse regimes);

- Representative maps of cellular phase state at the end (as opposed to peak in Fig 4) of excitation pulses of differing duration, and corresponding dependences of reflectivity on time.

\section{Acknowledgments}

This work was supported by the UK Engineering and Physical Sciences Research Council [grant EP/M009122/1], the Singapore Ministry of Education [grant MOE2016-T3-1-006], and the National 
Natural Science Foundation of China [grant U1804165]. The authors would like to thank E. T. F. Rogers, P. Cencillo-Abad, and V. Savinov for constructive inputs and discussion.

\section{Data Availability}

Data sharing is not applicable to this article as no new data were created in this study.

\section{References}

1. S. Raoux, Annu. Rev. Mater. Res. 39, 25 (2009).

2. M. Wuttig and N. Yamada, Nat. Mater. 6, 824 (2007).

3. J. Lawrence, Advances in Laser Materials Processing. (Woodhead Publishing, 2018).

4. M. Malinauskas, A. Žukauskas, S. Hasegawa, Y. Hayasaki, V. Mizeikis, R. Buividas and S. Juodkazis, Light Sci. Appl. 5, e16133 (2016).

5. K. Sugioka and Y. Cheng, Light Sci. Appl. 3, e149 (2014).

6. M. Mao, J. He, X. Li, B. Zhang, Q. Lei, Y. Liu and D. Li, Micromachines 8, 113 (2017).

7. D. Jin, Q. Chen, T.-Y. Huang, J. Huang, L. Zhang and H. Duan, Mater. Today 32, 19 (2020).

8. Y. Xie, D. J. Heath, J. A. Grant-Jacob, B. S. Mackay, M. D. T. McDonnell, M. Praeger, R. W. Eason and B. Mills, Journal of Physics: Photonics 1, 035002 (2019).

9. N. I. Zheludev, Nat. Photon. 1, 551 (2007).

10. K. J. Miller, R. F. Haglund and S. M. Weiss, Opt. Mater. Express 8, 2415 (2018).

11. A. M. Shaltout, V. M. Shalaev and M. L. Brongersma, Science 364, eaat3100 (2019).

12. M. Wuttig, H. Bhaskaran and T. Taubner, Nat. Photon. 11, 465 (2017).

13. B. J. Eggleton, B. Luther-Davies and K. Richardson, Nat. Photon. 5, 141 (2011).

14. F. Ding, Y. Yang and S. I. Bozhevolnyi, Adv. Optical Mater. 7, 1801709 (2019).

15. I. Chakraborty, A. Jaiswal, A. K. Saha, S. K. Gupta and K. Roy, Appl. Phys. Rev. 7, 021308 (2020).

16. T. Alexoudi, G. T. Kanellos and N. Pleros, Light Sci. Appl. 9, 91 (2020).

17. B. Gholipour, J. Zhang, K. F. MacDonald, D. W. Hewak and N. I. Zheludev, Adv. Mater. 25, 3050 (2013).

18. A. Karvounis, B. Gholipour, K. F. MacDonald and N. I. Zheludev, Appl. Phys. Lett. 109, 051103 (2016).

19. M. Rudé, R. E. Simpson, R. Quidant, V. Pruneri and J. Renger, ACS Photon. 2, 669 (2015).

20. M. Rudé, J. Pello, R. E. Simpson, J. Osmond, G. Roelkens, J. J. G. M. v. d. Tol and V. Pruneri, Appl. Phys. Lett. 103, 141119 (2013).

21. A. K. U. Michel, P. Zalden, D. N. Chigrin, M. Wuttig, A. M. Lindenberg and T. Taubner, ACS Photon. 1, 833 (2014).

22. A.-K. U. Michel, A. Heßler, S. Meyer, J. Pries, Y. Yu, T. Kalix, M. Lewin, J. Hanss, A. De Rose, T. W. W. Maß, M. Wuttig, D. N. Chigrin and T. Taubner, Adv. Mater. 31, 1901033 (2019). 
23. Q. Wang, J. Maddock, E. T. F. Rogers, T. Roy, C. Craig, K. F. MacDonald, D. W. Hewak and N. I. Zheludev, Appl. Phys. Lett. 104, 121105 (2014).

24. Q. Wang, E. T. F. Rogers, B. Gholipour, C. M. Wang, Y. Guanghui, J. Teng and N. I. Zheludev, Nat. Photon. 10, 60 (2016).

25. C. R. de Galarreta, A. M. Alexeev, Y.-Y. Au, M. Lopez-Garcia, M. Klemm, M. Cryan, J. Bertolotti and C. D. Wright, Adv. Funct. Mater. 28, 1704993 (2018).

26. J. Feldmann, N. Youngblood, C. D. Wright, H. Bhaskaran and W. H. P. Pernice, Nature 569, 208 (2019).

27. Y. Ke, S. Wang, G. Liu, M. Li, T. J. White and Y. Long, Small 14, 1802025 (2018).

28. M. F. Becker, A. B. Buckman, R. M. Walser, T. Lépine, P. Georges and A. Brun, Appl. Phys. Lett. 65, 1507 (1994).

29. A. Cavalleri, C. Toth, C. W. Siders, J. A. Squier, F. Raksi, P. Forget and J. C. Kieffer, Phys. Rev. Lett. 87, 237401 (2001).

30. J. Laverock, S. Kittiwatanakul, A. A. Zakharov, Y. R. Niu, B. Chen, S. A. Wolf, J. W. Lu and K. E. Smith, Phys. Rev. Lett. 113, 216402 (2014).

31. V. R. Morrison, R. P. Chatelain, K. L. Tiwari, A. Hendaoui, A. Bruhács, M. Chaker and B. J. Siwick, Science 346, 445 (2014).

32. Z. Tao, F. Zhou, T.-R. T. Han, D. Torres, T. Wang, N. Sepulveda, K. Chang, M. Young, R. R. Lunt and C.-Y. Ruan, Sci. Rep. 6, 38514 (2016).

33. M. R. Otto, L. P. René de Cotret, D. A. Valverde-Chavez, K. L. Tiwari, N. Émond, M. Chaker, D. G. Cooke and B. J. Siwick, Proc. Natl. Acad. Sci. 116, 450 (2019).

34. L. Vidas, D. Schick, E. Martínez, D. Perez-Salinas, A. Ramos-Álvarez, S. Cichy, S. Batlle-Porro, A. S. Johnson, K. A. Hallman, R. F. Haglund and S. Wall, Phys. Rev. X 10, 031047 (2020).

35. M. Rini, A. Cavalleri, R. W. Schoenlein, R. López, L. C. Feldman, R. F. Haglund Jr., L. A. Boatner and T. E. Haynes, Opt. Lett. 30, 558 (2005).

36. C. Kübler, H. Ehrke, R. Huber, R. Lopez, A. Halabica, R. F. Haglund and A. Leitenstorfer, Phys. Rev. Lett. 99, 116401 (2007).

37. H. W. Liu, L. M. Wong, S. J. Wang, S. H. Tang and X. H. Zhang, J. Phys.: Condens. Matter 24, 415604 (2012).

38. O. L. Muskens, L. Bergamini, Y. Wang, J. M. Gaskell, N. Zabala, C. H. de Groot, D. W. Sheel and J. Aizpurua, Light Sci. Appl. 5, e16173 (2016).

39. M. J. Dicken, K. Aydin, I. M. Pryce, L. A. Sweatlock, E. M. Boyd, S. Walavalkar, J. Ma and H. A. Atwater, Opt. Express 17, 18330 (2009).

40. T. Driscoll, H.-T. Kim, B.-G. Chae, B.-J. Kim, Y.-W. Lee, N. M. Jokerst, S. Palit, D. R. Smith, M. Di Ventra and D. N. Basov, Science 325, 1518 (2009).

41. R. M. Briggs, I. M. Pryce and H. A. Atwater, Opt. Express 18, 11192 (2010).

42. N. A. Butakov, I. Valmianski, T. Lewi, C. Urban, Z. Ren, A. A. Mikhailovsky, S. D. Wilson, I. K. Schuller and J. A. Schuller, ACS Photon. 5, 371 (2018).

43. J. Rensberg, S. Zhang, Y. Zhou, A. S. McLeod, C. Schwarz, M. Goldflam, M. Liu, J. Kerbusch, R. Nawrodt, S. Ramanathan, D. N. Basov, F. Capasso, C. Ronning and M. A. Kats, Nano Lett. 16, 1050 (2016). 
44. D. Y. Lei, K. Appavoo, F. Ligmajer, Y. Sonnefraud, R. F. Haglund Jr. and S. A. Maier, ACS Photon. 2, 1306 (2015).

45. C. Wan, E. H. Horak, J. King, J. Salman, Z. Zhang, Y. Zhou, P. Roney, B. Gundlach, S. Ramanathan, R. H. Goldsmith and M. A. Kats, ACS Photon. 5, 2688 (2018).

46. P. J. Bennett, S. Dhanjal, P. Petropoulos, D. J. Richardson, N. I. Zheludev and V. I. Emel'yanov, Appl. Phys. Lett. 73, 1787 (1998).

47. P. Petropoulos, H. L. Offerhaus, D. J. Richardson, S. Dhanjal and N. I. Zheludev, Appl. Phys. Lett. 74, 3619 (1999).

48. R. F. Waters, P. A. Hobson, K. F. MacDonald and N. I. Zheludev, Appl. Phys. Lett. 107, 081102 (2015).

49. A. V. Krasavin and N. I. Zheludev, Appl. Phys. Lett. 84, 1416 (2004).

50. A. V. Krasavin, K. F. MacDonald, N. I. Zheludev and A. V. Zayats, Appl. Phys. Lett. 85, 3369 (2004).

51. S. R. C. Vivekchand, C. J. Engel, S. M. Lubin, M. G. Blaber, W. Zhou, J. Y. Suh, G. C. Schatz and T. W. Odom, Nano Lett. 12, 4324-4328 (2012).

52. B. F. Soares, K. F. MacDonald, V. A. Fedotov and N. I. Zheludev, Nano Lett. 5, 2104 (2005).

53. A. I. Denisyuk, K. F. MacDonald, F. J. García de Abajo and N. I. Zheludev, Jpn. J. Appl. Phys. 48, 03 A065 (2009).

54. Y. Gutiérrez, M. Losurdo, P. García-Fernández, M. Sainz de la Maza, F. González, A. S. Brown, H. O. Everitt, J. Junquera and F. Moreno, Opt. Mater. Express 9, 4050 (2019).

55. Y. Gutiérrez, M. Losurdo, P. García-Fernández, M. Sainz de la Maza, F. González, A. S. Brown, H. O. Everitt, J. Junquera and F. Moreno, Adv. Optical Mater. 7, 1900307 (2019).

56. J. Hegedüs and S. Elliott, Nat. Mater. 7, 399 (2008).

57. T. H. Lee and S. R. Elliott, Phys. Rev. Lett. 107, 145702 (2011).

58. S. Senkader and C. D. Wright, J. Appl. Phys. 95, 504 (2004).

59. J. Scoggin, Z. Woods, H. Silva and A. Gokirmak, Appl. Phys. Lett. 114, 043502 (2019).

60. F. Tabatabaei, G. Boussinot, R. Spatschek, E. A. Brener and M. Apel, J. Appl. Phys. 122, 045108 (2017).

61. S. Meyer, Z. Y. Tan and D. N. Chigrin, Nanophotonics 9, 675 (2020).

62. U. Russo, D. Ielmini, A. Redaelli and A. L. Lacaita, IEEE Transactions on Electron Devices 53, 3032 (2006).

63. X. Ao, H. Xia, J. Liu and Q. He, Mater. Design 185, 108230 (2020).

64. Q. Zhang, H. Xue, Q. Tang, S. Pan, M. Rettenmayr and M. Zhu, Comp. Mater. Sci. 146, 204 (2018).

65. K. Reuther and M. Rettenmayr, Comp. Mater. Sci. 95, 213 (2014).

66. L. Bai, B. Wang, H. Zhong, J. Ni, Q. Zhai and J. Zhang, Metals 6, 53 (2016).

67. P. Ashwin, B. S. V. Patnaik and C. D. Wright, J. Appl. Phys. 104, 084901 (2008).

68. C. Mihai and A. Velea, Model. Simul. Mater. Sc. 26, 045006 (2018).

69. J. L. Guisado, F. Jimenez-Morales and J. M. Guerra, Phys. Rev. E 67, 066708 (2003). 
70. J. G. Kelly and E. C. Boyer, Cryst Growth Des 14, 1392 (2014).

71. G. H. B. Miranda, J. Machicao and O. M. Bruno, Sci. Rep. 6, 37329 (2016).

72. Y. Wang, M. An, Z. Yu, B. Han and W. Ji, Constr. Build Mater. 172, 760 (2018).

73. F. Centrone, C. Tassi, M. Barbieri and A. Serafini, Phys. Rev. A 98, 012105 (2018).

74. A. Bisio, G. M. D’Ariano and P. Perinotti, Ann. Phys. - New York 368, 177 (2016).

75. V. S. Kalogeiton, D. P. Papadopoulos, O. Liolis, V. A. Mardiris, G. C. Sirakoulis and I. G. Karafyllidis, IEEE T. Comput. Aid. D. 36, 1367 (2017).

76. J. Li and T. C. H. Liew, Opt. Express 24, 24930 (2016).

77. F. Perez-Martinez, K. D. Petersson, I. Farrer, D. Anderson, G. A. C. Jones, D. A. Ritchie and C. G. Smith, Microelectr. J. 39, 674 (2008).

78. P. Chavel, J. Taboury, F. Devos and P. Garda, J. Phys. - Paris 49, C2_35 (1988).

79. J. F. Botía, A. M. Cárdenas and C. M. Sierra, Eng. Appl. Artif. Intel. 62, 181 (2017).

80. F. Previdi and M. Milani, Nuovo Cimento D 11, 1625 (1998).

81. C. Conti, in Game of Life Cellular Automata, edited by A. Adamtzky (Springer, London, 2010).

82. N. R. Comins, Phil. Mag. 25, 817 (1972).

83. O. Hunderi and R. Ryberg, J. Phys. F Met. Phys. 4, 2096 (1974).

84. M. Bernasconi, G. L. Chiarotti and E. Tosatti, Phys. Rev. B 52, 9988 (1995).

85. R. Kofman, P. Cheyssac and J. Richard, Phys. Rev. B 16, 5216 (1977).

86. E. Voloshina, K. Rosciszewski and B. Paulus, Phys. Rev. B 79, 045113 (2009).

87. R. Trittibach, C. Grutter and J. H. Bilgram, Phys. Rev. B 50, 2529 (1994).

88. V. Albanis, S. Dhanjal, V. A. Fedotov, K. F. MacDonald, N. I. Zheludev, P. Petropoulos, D. J. Richardson and V. I. Emel'yanov, Phys. Rev. B 63, 165207 (2001).

89. A. V. Rode, M. Samoc, B. Luther-Davies, E. G. Gamaly, K. F. MacDonald and N. I. Zheludev, Opt. Lett. 26, 441 (2001).

90. O. P. Uteza, E. G. Gamaly, A. V. Rode, M. Samoc and B. Luther-Davies, Phys. Rev. B 70, 054108 (2004).

91. B. Chopard and M. Droz, Cellular Automata Modeling of Physical Systems. (Cambridge University Press, 1998).

92. S. Wolfram, Cellular Automata and Complexity: Collected Papers. (CRC Press, 1994).

93. V. P. Zhukov and E. V. Chulkov, Phys-Usp+ 52, 105 (2009).

94. V. Heine, J. Phys. C (Proc. Phys. Soc.) 1, 222 (1968).

95. J. T. Schriempf, Solid State Commun. 13, 651 (1973).

96. M. E. Povarnitsyn, T. E. Itina, K. V. Khishchenko and P. R. Levashov, Appl. Surf. Sci. 253, 6343 (2007). 


\section{Supplementary Material:}

\section{Cellular Automata Dynamics of Nonlinear Optical Processes in a Phase-change Material}

Liwei Zhang $^{1,2}$, Robin F. Waters ${ }^{1}$, Kevin F. MacDonald ${ }^{1}$, and Nikolay I. Zheludev ${ }^{1,3}$

${ }^{1}$ Optoelectronics Research Centre \& Centre for Photonic Metamaterials, University of Southampton, Highfield, Southampton, SO17 1BJ, UK

${ }^{2}$ School of Mathematics and Physics, Anqing Normal University, Anqing 246133, P. R. China

${ }^{3}$ Centre for Disruptive Photonic Technologies \& The Photonics Institute, School of Physical and Mathematical Sciences, Nanyang Technological University, Singapore, 637371

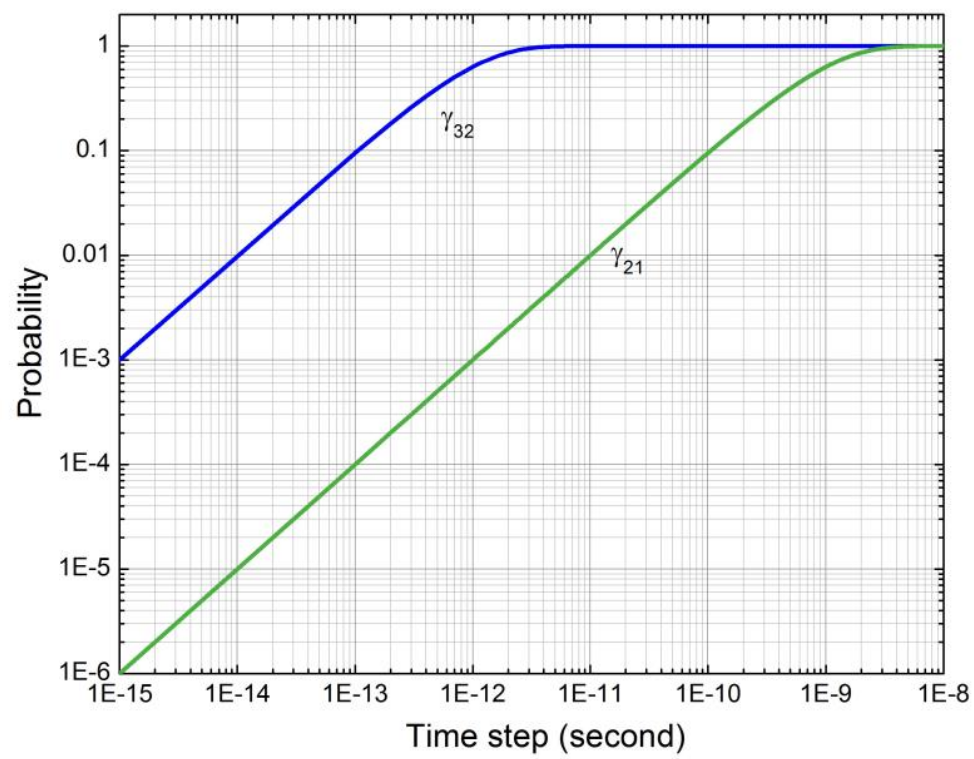

Fig. S1. Dependences of transition probabilities $\gamma_{32}$ [from optically excited to metallic state] and $\gamma_{21}$ [from metallic to ground state] on CA model time step $\delta t: \gamma_{m n}=1-e^{-\delta t / \tau_{m}}$, where $\tau_{m}$ is the lifetime of the upper state $\left[\tau_{3}=1 \mathrm{ps} ; \tau_{2}=1 \mathrm{~ns}\right]$. 


\section{Photon absorption probability per cell}

The familiar expression for the dependence of light intensity on propagation distance $x$ within a continuous medium

$$
I_{x}=I_{0} e^{-2 x / \delta}
$$

where $I_{0}$ is the incident intensity and $\delta$ is the optical skin depth, can be cast in a form for propagation through $n$ identical, discrete but contiguous layers of material

$$
I_{n}=I_{0}(1-A b)^{n}
$$

where $A b$ is the probability of a photon being absorbed in a single layer.

From the equivalence of these expressions

$$
A b=1-e^{-2 \Delta x / \delta}
$$

where $\Delta x$ is the thickness of each layer (in the propagation direction).

In the present Ga CA model, cell size $\Delta x=0.55 \mathrm{~nm}$ and the skin depth (of the ground state $\alpha$-phase at a wavelength of $755 \mathrm{~nm}$ ) $\delta=37 \mathrm{~nm}$, giving an absorption probability per cell $A b=0.03$. 


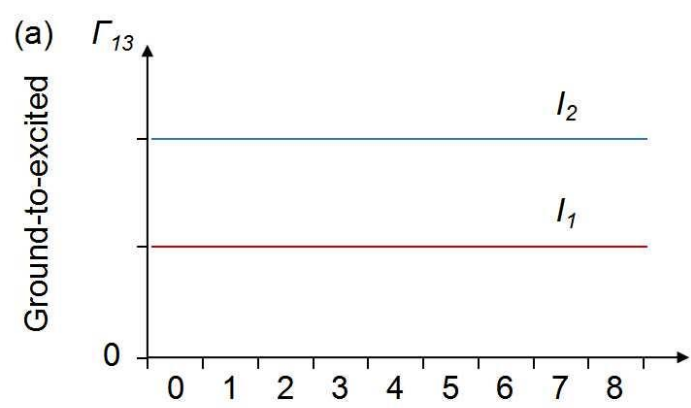

Neighbours in excited/metallic state

(c)

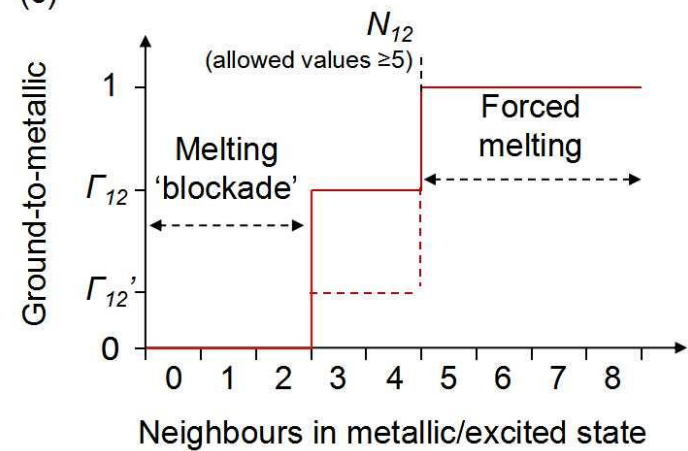

(b)

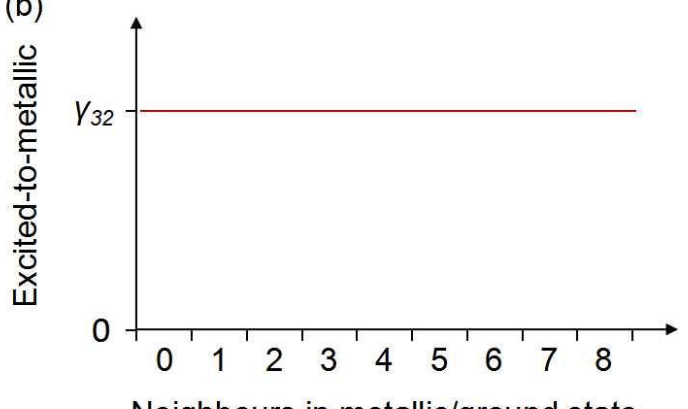

Neighbours in metallic/ground state

(d)

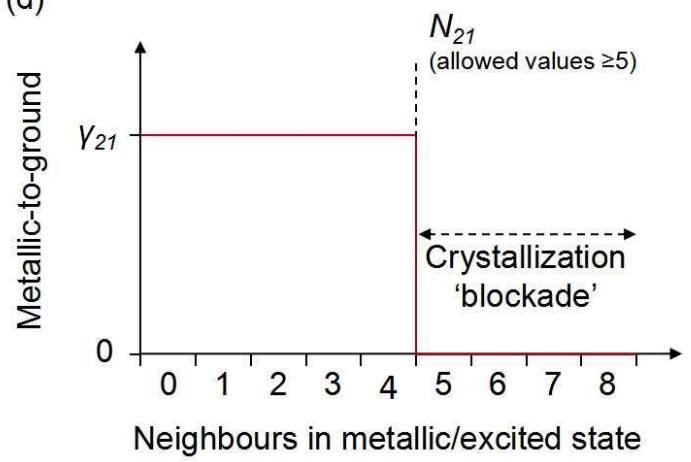

Fig. S2. Schematic dependences of transition probabilities for a given cell on its neighborhood - the phase state of the surrounding eight [nearest and next-nearest] cells. (a) Ground to excited state transitions via absorption of a photon leading to breaking of a Ga dimer bond. Probability is independent of neighboring cell state; value $\Gamma_{13}$ is proportional to incident intensity, i.e. photon flux. (b) Excited to metallic state decay. Probability is independent of neighboring cell state; value $\gamma_{32}$ is given by excited state lifetime $\tau_{3}$. (c) Thermally driven ground to metallic state excitation. Probability is dependent upon neighboring cell state; values $\Gamma_{12}$ and $\Gamma_{12}{ }^{\prime}$ are functions of local temperature and apply respectively in the presence and absence of optical excitation. [ $\Gamma_{12}$ ' must be less than $\gamma_{21}$ for the avoidance of spontaneous metallization at $T<T_{m}$.] (d) Metallic to ground state decay. Probability is dependent upon neighboring cell state; value $\gamma_{21}$ below the $N_{21}$ blockade threshold is given by excited state lifetime $\tau_{2}$. 


\section{Threshold value of $\Gamma_{12}$}

The probability $\Gamma_{12}$ of thermally driven ground to metallic state excitation represents local temperature within gallium cells, i.e. ambient plus a contribution due to optically-induced heating. In experimental practice there is a threshold ambient temperature below which (a maximum value of $T_{m}-T$ above which) a given excitation fluence will not induce an observable change in reflectivity. Correspondingly, in the CA model, for a given incident fluence $F_{\text {in }}$ and set of neighbor thresholds $N_{12}$, 21 and state lifetimes $\tau_{2,3}$, there is a threshold value of $\Gamma_{12}$ below which no excitation-induced reflectivity change is seen.

This threshold can be empirically evaluated using the CA model (Fig. S3) and, as one may expect, in the long-pulse regime dominated by thermally-driven transitions it is found to track the dependence of $\gamma_{2 l}$ on decreasing time step $\delta t$ or rather pulse duration $\tau_{p}(=100 \delta t)$. The trend is then offset in the short pulse regime through a shoulder in the curve at $\tau_{p} \sim \tau_{3}$. It is notable here that this shoulder occurs at a much shorter timescale than the observed transition between thermally- and non-thermallydominated reflectivity dynamics (at $\tau_{p} \sim 10 \mathrm{~ns}$ - see Figs. 4 and S6), and moreover that the threshold value of a parameter relating to transitions between states 1 (ground) and 2 (metallic) is apparently dependent on the lifetime of state 3 (optically excited). Both of these behaviors may be accounted for by a combination of the fact that while it can only be populated directly from the ground state (1), relaxation of the optically excited state (3) occurs via the metallic state (2), and that the metallic state (2) is populated from both the ground (1) and excited (3) states.

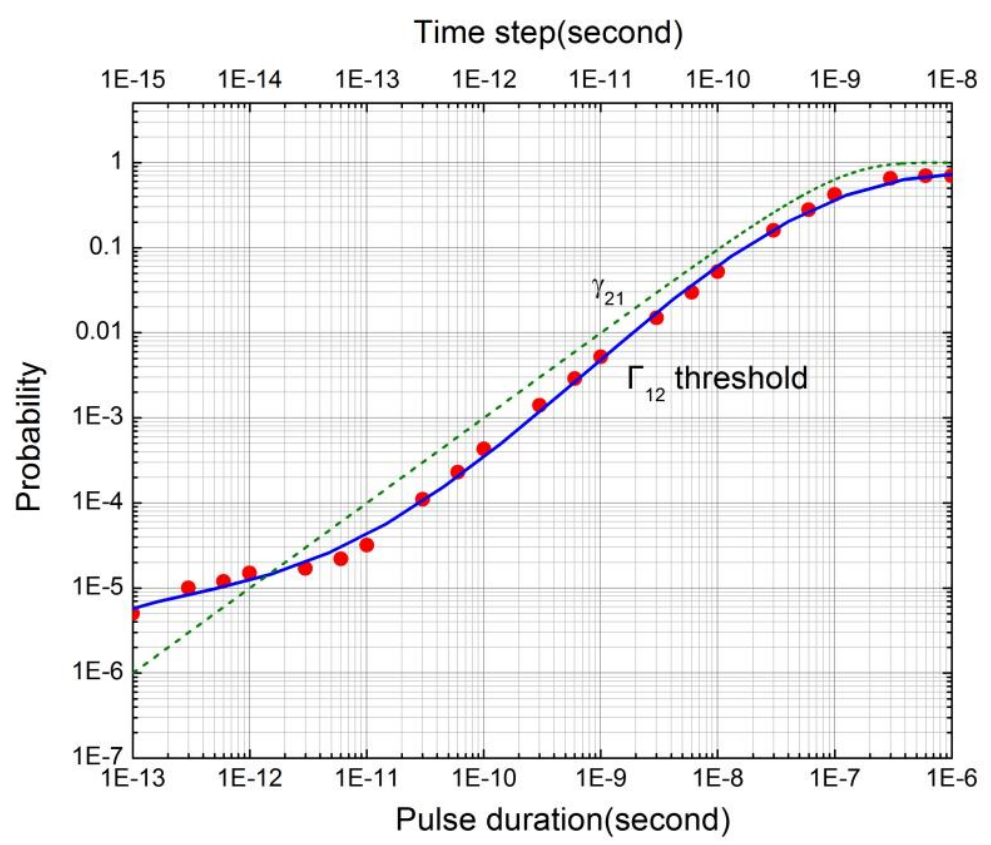

Fig. S3. Empirically evaluated dependence of $\Gamma_{12}$ threshold on pulse duration $\left[F_{i n}=5 \mathrm{~mJ} / \mathrm{cm}^{2} ; N_{12}, N_{21}=5\right]$. 

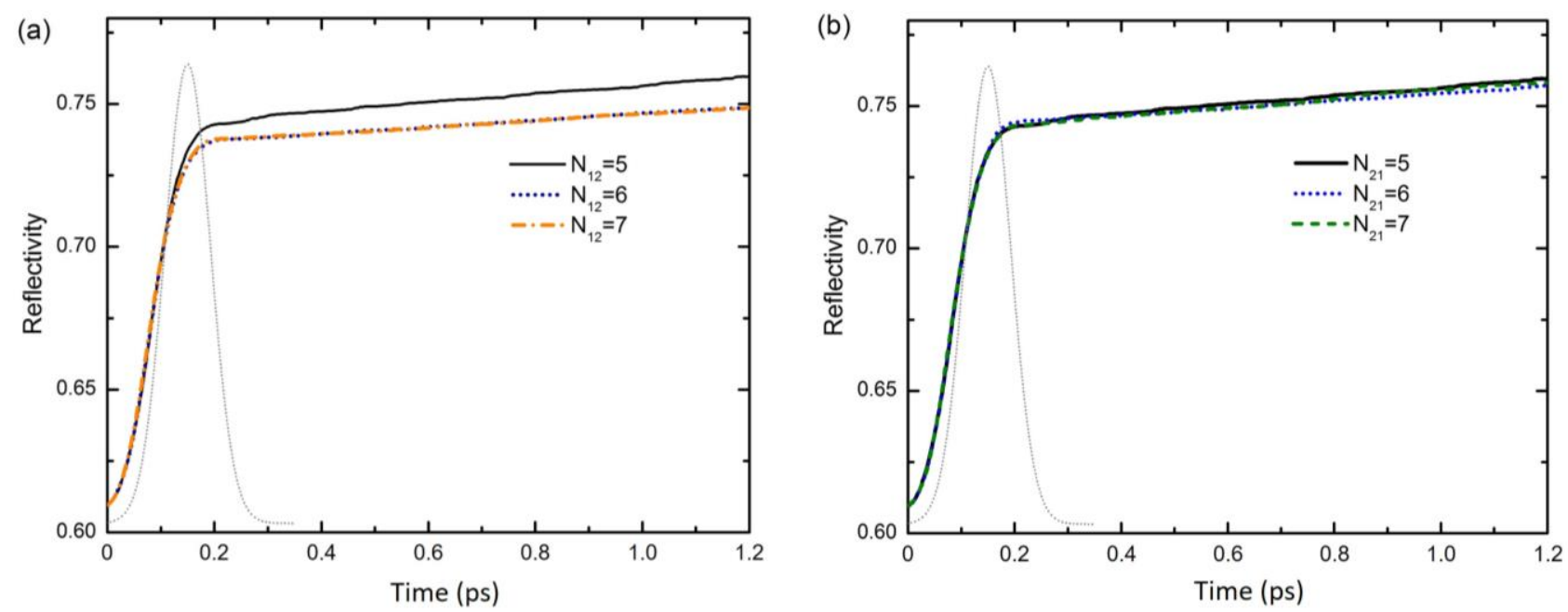

Fig. S4. Effect of neighbor threshold values $N_{21}$ and $N_{12}$ on reflectivity dynamics in the short pulse excitation regime [pulse duration $\tau_{p}=100 \mathrm{fs}$; time step $\delta t=1 \mathrm{fs} ; F_{i n}=5 \mathrm{~mJ} / \mathrm{cm}^{2} ; \Gamma_{12}=8 \times 10^{-5}$ ]. In (a) and (b) respectively $N_{12}$ and $N_{21}$ have fixed values of 5 . [The black $N_{12}, N_{21}=5$ curves here correspond to the black $5 \mathrm{~mJ} / \mathrm{cm}^{2}$ curve in Fig. 2a.] 
(a)

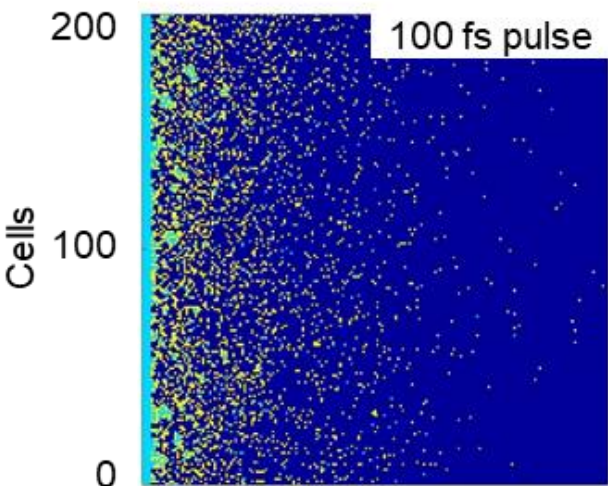

(c)

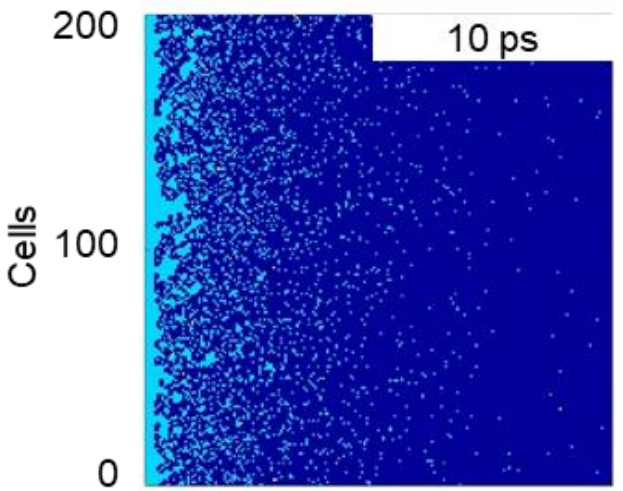

(e)

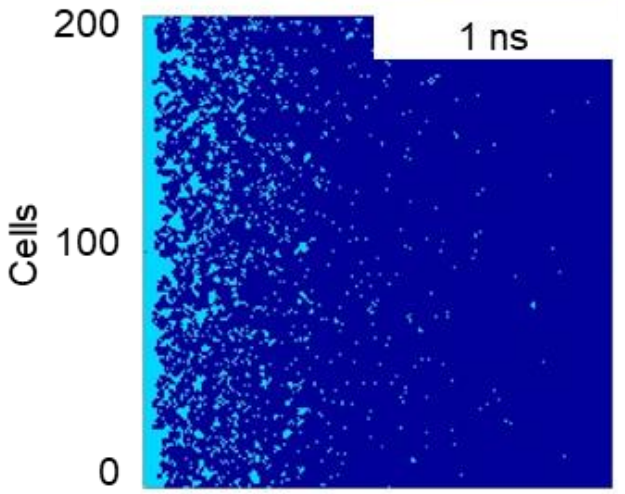

(g)

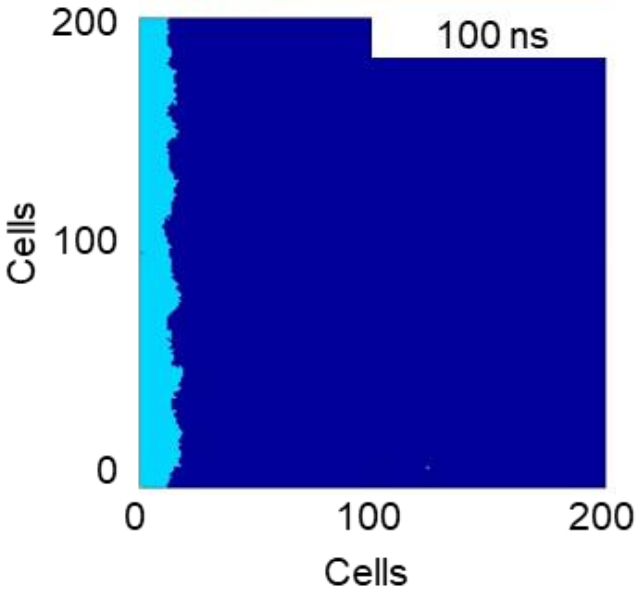

(b)

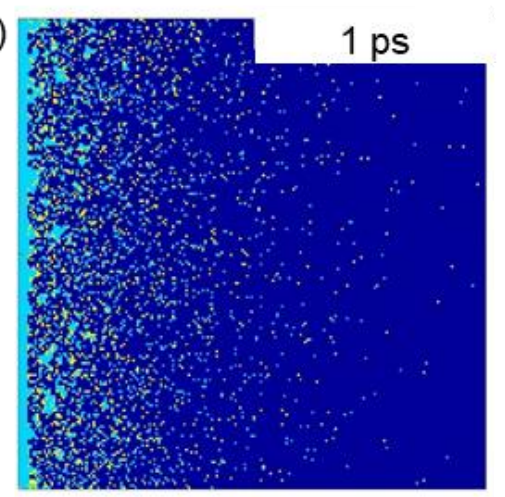

(d)

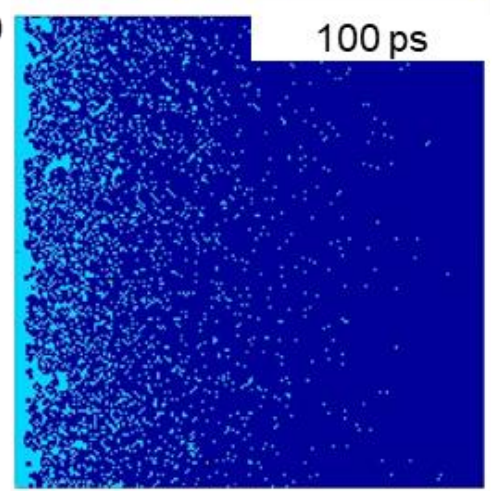

(f)

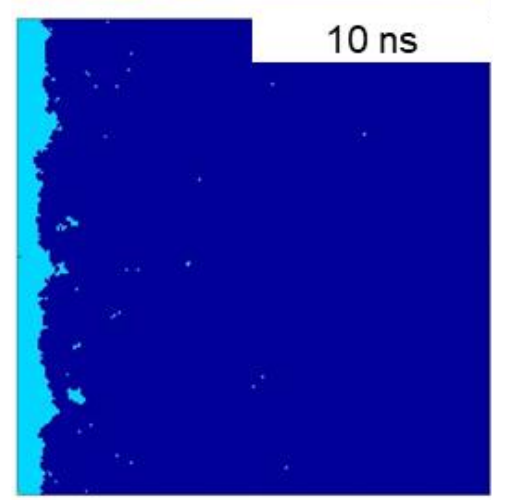

(h)

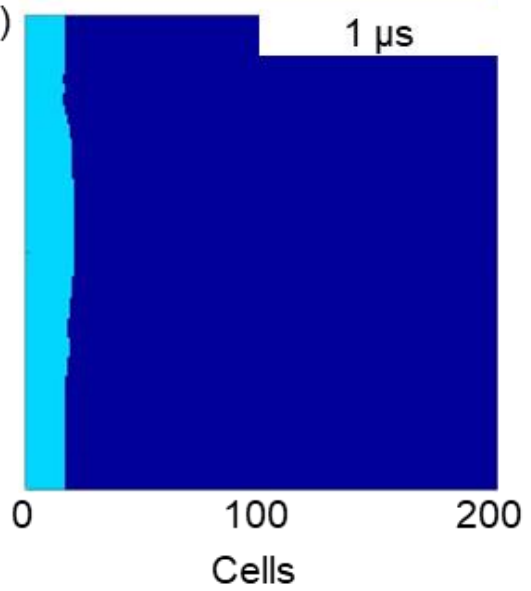

Ground

Optically

excited

Metallic

- 10 nm

Fig. S5. Representative maps of cellular phase state at the tail end [as compared to peak shown in Fig 4] of Gaussian excitation pulses with durations $\tau_{p}$ ranging from $100 \mathrm{fs}$ to $1 \mu \mathrm{s}$ [as labelled] in order-of-magnitude increments. Each frame is taken at an interval after the peak equal to $\tau_{p}$ - i.e. $100 \mathrm{fs}$ after the peak of a $100 \mathrm{fs}$ pulse, 1 ps after the peak of a 1 ps pulse, etc. [All model parameters as per Fig. 4]. 

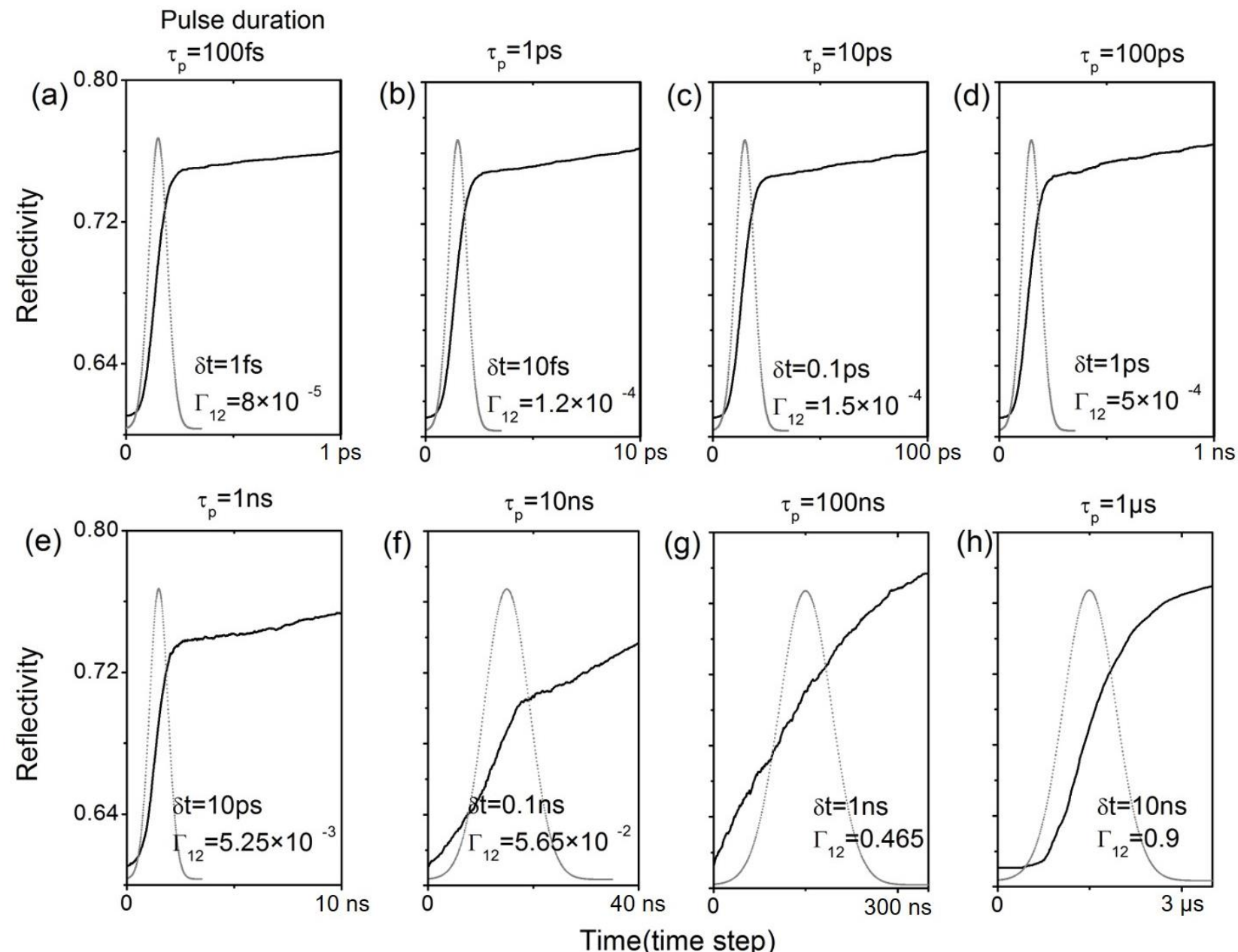

Fig. S6. Reflectivity dynamics as a function of pulse duration. CA modelled Ga-silica interface reflectivity at $775 \mathrm{~nm}$ as a function of time for Gaussian excitation pulses with durations $\tau_{p}$ ranging from $100 \mathrm{fs}$ to $1 \mu \mathrm{s}$ [as labelled] in order-of-magnitude increments. [ $F_{\text {in }}=5 \mathrm{~mJ} / \mathrm{cm}^{2} ; N_{12}, N_{21}=5$; values of $\Gamma_{12}$ are shown inset.] The dashed grey line shows the temporal profile of the excitation pulse [normalized amplitude - vertical axis scale does not apply]. Note that shorter relative temporal ranges are plotted in (f)-(h) where $\tau_{p} \rightarrow \tau_{t}$ and the assumption that $\Gamma_{12}$ holds a constant value beyond the duration of the pulse is no longer valid. 

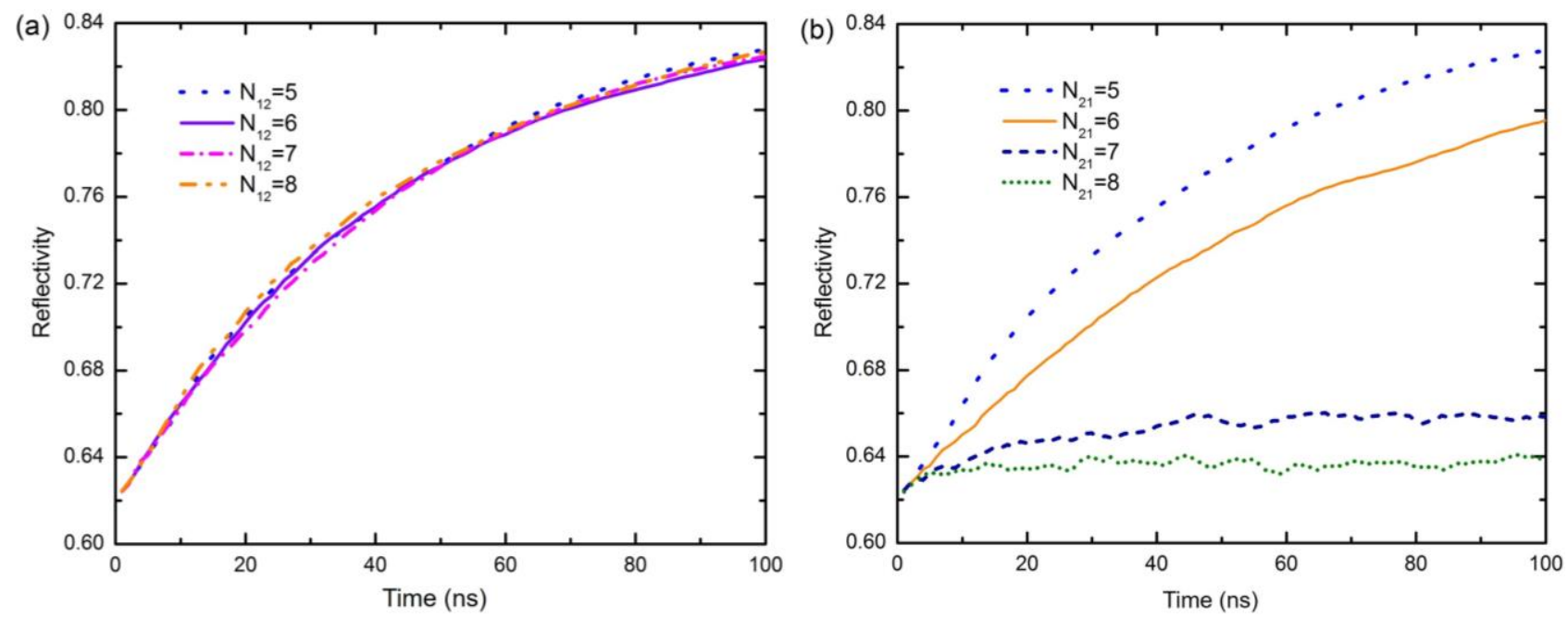

Fig. S7. Effect of neighbor threshold values $N_{21}$ and $N_{12}$ on reflectivity dynamics in the long pulse excitation regime [pulse duration $\tau_{p}=100 \mathrm{~ns}$; time step $\delta t=1 \mathrm{~ns} ; \Gamma_{12}=0.8$. In (a) and (b) respectively $N_{12}$ and $N_{21}$ have fixed values of 5. [The dashed blue $N_{12}, N_{21}=5$ curves here correspond to the dashed blue $\Gamma_{12}=0.8$ curve in Fig. 5a.] 